\title{
Signature of heavy sterile neutrinos at CEPC
}

\author{
Wei Liao and Xiao-Hong Wu \\ Institute of Modern Physics, School of Science, East China University of Science and Technology, \\ Meilong Road 130, Shanghai 200237, China
}

(Received 31 October 2017; published 5 March 2018)

\begin{abstract}
We study the production of heavy sterile neutrino $N, e^{+} e^{-} \rightarrow N \nu(\bar{\nu})$, at the Circular Electron Positron Collider (CEPC) and its $l j j$ signal in its decay to three charged fermions. We study background events for this process which are mainly events coming from $\mathrm{W}$ pair production. We study the production of a single heavy sterile neutrino and the sensitivity of CEPC to the mixing of the sterile neutrino with active neutrinos. We study the production of two degenerate heavy sterile neutrinos in a low energy seesaw model by taking into account the constraints on mixings of sterile neutrinos from the neutrinoless double $\beta$ decay experiment and the masses and mixings of active neutrinos. We show that CEPC under proposal has a good sensitivity to the mixing of sterile neutrinos with active neutrinos for a mass of a sterile neutrino around $100 \mathrm{GeV}$.
\end{abstract}

DOI: 10.1103/PhysRevD.97.055005

\section{INTRODUCTION}

The establishment of neutrino oscillation and tiny masses of active neutrinos in past decades has raised strong hope that new physics beyond the Standard Model (SM) may exist in the leptonic sector of elementary particles. The seesaw mechanism [1], as a simple and straightforward extension of neutrinos in the SM, works as a very good mechanism to explain the tiny masses of active neutrinos and is a very good candidate for physics beyond the SM. In the seesaw mechanism, several right-handed neutrinos uncharged under the SM gauge groups, hence a type of sterile neutrino, are introduced with heavy Majorana-type masses which violate lepton number. The tiny masses of active neutrinos are understood in the low energy scale as the lepton number violating remnant of the Majorana-type masses of heavy right-handed neutrinos.

Although seesaw-type models are quite interesting models of physics beyond the SM and have fruitful implications, there are very few clues regarding the mass scale of right-handed neutrinos. In particular, the mass scale of right-handed neutrinos can be much higher than the electroweak scale. Therefore, it is very hard to test models of such type in experiments if such a hierarchy between the mass scale of right-handed neutrinos and the electroweak scale indeed exists. For this reason, a low energy scale seesaw type model [2], which has right-handed neutrinos at

Published by the American Physical Society under the terms of the Creative Commons Attribution 4.0 International license. Further distribution of this work must maintain attribution to the author(s) and the published article's title, journal citation, and DOI. Funded by SCOAP ${ }^{3}$. or below the electroweak scale, is quite interesting since it is possible to test it in experiments. There are several interesting properties of this low energy seesaw model. For example, one of the right-handed neutrinos can be of $\mathrm{keV}$ scale and serves as a good candidate for warm dark matter (WDM) in the universe. Two other right-handed neutrinos in the model are at $\mathrm{GeV}$ or $100 \mathrm{GeV}$ scale and are sufficient to generate tiny masses and mixings of active neutrinos measured in neutrino oscillation experiments.

Another interesting property in this type of low energy seesaw model is that the Yukawa couplings of right-handed neutrinos with SM neutrinos can be quite large while still giving rise to masses and mixing of active neutrinos consistent with the experimental data in neutrino oscillation and the constraint from neutrinoless double $\beta(0 \nu \beta \beta)$ experiment $[3,4]$, in particular when two heavy righthanded neutrinos are degenerate or quasidegenerate. Consequently, the mixings of right-handed neutrinos with active neutrinos in the SM can be quite large when the masses of right-handed neutrinos are at $\mathrm{GeV}$ to $100 \mathrm{GeV}$ scale. This scenario apparently offers great opportunities to search for seesaw-type models of physics beyond the SM in collider experiments.

Experimentally, the single heavy neutrino has been searched for by the L3 collaboration at LEP through the $N \rightarrow e W$ channel [5,6]. A stringent constraint on $\left|R_{e N}\right|^{2}$ has been set for a mass region from $80 \mathrm{GeV}$ to $205 \mathrm{GeV}$. Some efforts have been made to study the production and signature of heavy neutrinos in $e^{+} e^{-}$or $e^{-} e^{-}$collision processes with both pair and single heavy neutrino productions, and various neutrino decay chains, $l W, \nu Z$ and $\nu H$ [7-26]; for a review, see [24]. Currently, new electronpositron colliders, such as CEPC, Future Circular Collider 
(FCC), and International Linear Collider (ILC), are under proposal. With these colliders, heavy sterile neutrinos can be probed to a larger mass range and with better sensitivity on the active-sterile mixing $R_{l N}$. Recently, single heavy neutrino production modes $N \nu$ and $N e^{ \pm} W^{\mp}$ at ILC with center of mass energy $350 \mathrm{GeV}$ and $500 \mathrm{GeV}$ have been investigated in [21]. A search of long-lived heavy neutrinos with displaced vertices at CEPC, FCC, and ILC has been presented in Ref. [23]. In our work, we present a detailed study of $e^{+} e^{-} \rightarrow N \nu$ with charged current neutrino decay mode $N \rightarrow l W$ at CEPC with center of mass energy $\sqrt{s}=240 \mathrm{GeV}$.

In the present article, we are motivated by such kinds of possibility and study the signature of this type of right-handed neutrino (or sterile neutrino) of $100 \mathrm{GeV}$ masses at CEPC [27], a collider under proposal. In the next section, we will make a quick review of the low energy seesaw model and describe some basic properties of this model. Then we discuss the collider signatures of a single sterile neutrino of a mass around $100 \mathrm{GeV}$. For simplicity, we simplify our discussion of collider signature using a single sterile neutrino. We will show that this simplification can be taken as a good simplification for later discussion. Then we come to signatures of the low energy seesaw model by including detailed constraints on the masses and mixings of right-handed neutrinos. We conclude in the last section.

\section{GEV SCALE STERILE NEUTRINO AND LOW ENERGY SEESAW MODEL}

One of major differences between the case of a single $\mathrm{GeV}$ scale sterile neutrino and the low energy seesaw-type model of $\mathrm{GeV}$ scale sterile neutrinos is that for the former the mixings of sterile neutrinos with active neutrino are strongly constrained by the $0 \nu \beta \beta$ decay experiment [28], while for the latter the $0 \nu \beta \beta$ constraint can be quite weak and the mixings can be quite large [3].

In the presence of one or several sterile neutrinos, active neutrinos in the flavor base $\nu_{l}(l=e, \mu, \tau)$ are a mixture of the light neutrinos in mass eigenstates $\nu_{i}(i=1,2,3)$ and heavy sterile neutrinos in mass eigenstates $N_{j}$,

$$
\nu_{l}=\sum_{i} U_{l i} \nu_{i}+\sum_{j} R_{l N_{j}} N_{j}
$$

where $U_{l j}$ is the Pontecorvo-Maki-Nakagawa-Sakata (PMNS) mixing matrix, and $R_{l N_{j}}$ is the matrix element mixing $\nu_{l}$ with heavy neutrinos $N_{j}$. For small enough $\left|R_{I N_{j}}\right|$, mixing matrix $U$ can be considered as approximately unitary. Apparently, $\nu_{i}$ and $N_{j}$ can all contribute, in a virtual intermediate state, to the $0 \nu \beta \beta$ decay. It is not hard to see that the contribution of a single $\mathrm{GeV}$ scale sterile neutrino to the amplitude of the $0 \nu \beta \beta$ decay is proportional to $R_{e N}^{2} / M_{N}$. The mixing $R_{e N}$ in this case is constrained to be
$\left|R_{e N}\right|^{2} \lesssim 10^{-5}$ [28], unless there are other particles or mechanisms at hand to ease the constraint.

In the low energy seesaw type model, at least two heavy sterile neutrinos (right-handed neutrinos) are needed to obtain the correct masses and flavor mixings of active neutrinos [3]. In this case, the mixing matrix $R$ is $R=$ $Y v\left(M^{*}\right)^{-1}$ where $Y$ is the Yukawa coupling of neutrinos, $\mathrm{v}$ the vacuum expectation value in the SM, and $M$ the Majorana mass matrix of sterile neutrinos which can be taken to be real and diagonal in a convenient base. The matrix $M$ is a $2 \times 2$ matrix if considering two heavy sterile neutrinos and a $3 \times 3$ matrix if considering three heavy sterile neutrinos.

A nice feature in the seesaw model is that mixing $R$ is related to $m_{\nu}$, the mass matrix of active neutrinos responsible for the neutrino oscillation phenomena,

$$
\left(m_{\nu}\right)_{l l^{\prime}}=-v^{2} \sum_{i} Y_{l i}^{*} Y_{l^{\prime} i}^{*} M_{i}^{-1}=-\sum_{i} M_{i} R_{l N_{i}}^{*} R_{l^{\prime} N_{i}}^{*}
$$

where $M_{i}$ is the eigenvalue of matrix $M$, that is, we have chosen a base in which $M$ is diagonal. One can see that if a strong cancellation happens between contributions of different sterile neutrinos in (2), a mass matrix $m_{\nu}$ at $10^{-3}-10^{-2} \mathrm{eV}$ scale can be generated for $M_{i}$ of $100 \mathrm{GeV}$ scale and for pretty large $\left|R_{l N_{i}}\right|$.

Using mixing matrix $R$, contributions of heavy sterile neutrinos to the amplitude of $0 \nu \beta \beta$ decay can be parametrized as follows:

$$
\mathcal{A}=F \sum_{i} R_{e N_{i}}^{2} M_{i}^{-1},
$$

where $F$ is an overall factor. For two heavy sterile neutrinos $N_{1}$ and $N_{2}$, (3) can be rewritten as

$\mathcal{A}=\frac{F}{M_{1}^{2}}\left(R_{e N_{1}}^{2} M_{1}+R_{e N_{2}}^{2} M_{2}\right)+F M_{2} R_{e N_{2}}^{2}\left(\frac{1}{M_{2}^{2}}-\frac{1}{M_{1}^{2}}\right)$,

where $M_{1}$ and $M_{2}$ are the masses of $N_{1}$ and $N_{2}$, respectively. By taking $M_{1,2}$ to be real in a convenient base, one can see in (2) and (4) that the first term in (4) is of order $10^{-3}-10^{-2} \mathrm{eV} / M_{1}^{2}$ and can be neglected. The second term in (4) can be arbitrarily small if $N_{1}$ and $N_{2}$ are quasidegenerate or degenerate. One can see clearly that the constraint from $0 \nu \beta \beta$ decay is no longer strong for two quasidegenerate heavy sterile neutrinos, which is exactly what happens in the low energy seesaw model.

A straightforward consequence of the above discussion about (4) and (2) and the degeneracy of $N_{1}$ and $N_{2}$ is that for sterile neutrinos of $\mathrm{GeV}$ to $100 \mathrm{GeV}$ mass, a large value of $\left|R_{e N_{i}}\right|^{2}$ is only possible when

$$
R_{e N_{1}}^{2}=-R_{e N_{2}}^{2}, \quad \text { or } \quad R_{e N_{1}}= \pm i R_{e N_{2}} .
$$



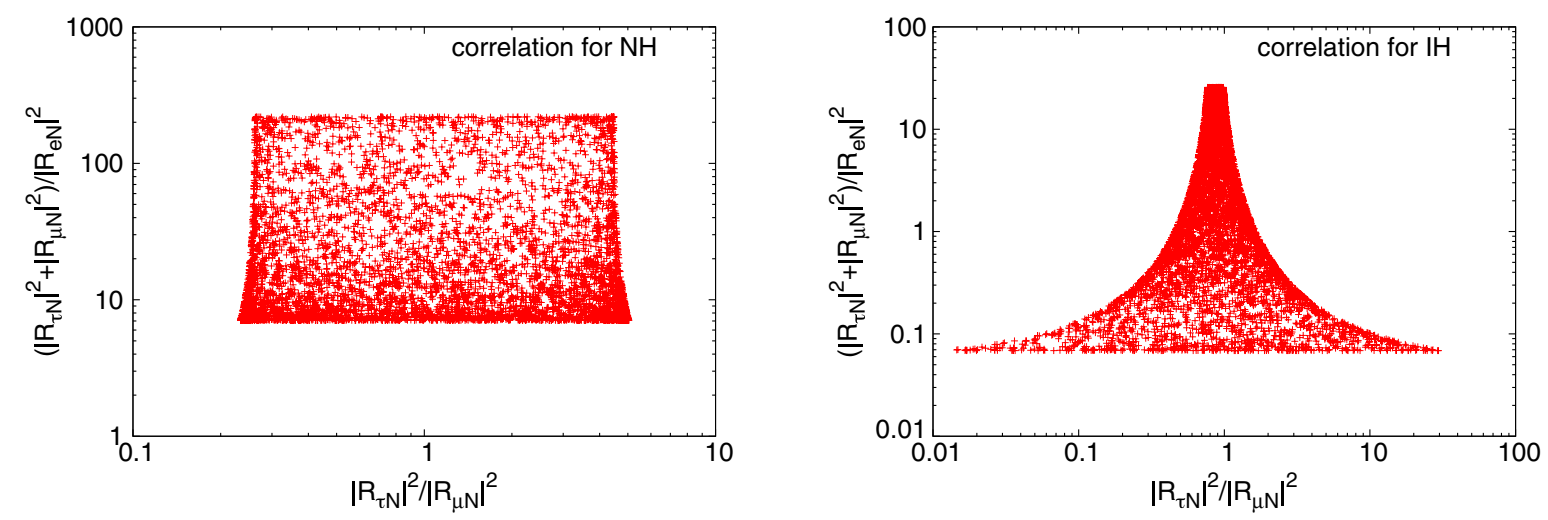

FIG. 1. $\quad\left(\left|R_{\tau N_{1}}\right|^{2}+\left|R_{\mu N_{1}}\right|^{2}\right) /\left|R_{e N_{1}}\right|^{2}$ versus $\left|R_{\tau N_{1}}\right|^{2} /\left|R_{\mu N_{1}}\right|^{2}$ for NH and IH, respectively.

Equation (5) is one of the major relations to be used in later analysis for discussing the collider signal of the low energy seesaw model.

Relations among $R_{\mu N_{i}}$ and $R_{\tau N_{i}}$ can also be addressed similarly. Using solutions presented for two heavy sterile neutrinos in [3], one can find that $R_{l N_{i}}$ can be expressed as

$$
\begin{aligned}
R_{l N_{1}}= & \frac{1}{2} e^{\mp i x+|y|}\left(U_{l 2} m_{2}^{1 / 2} e^{-i \phi_{2} / 2} \mp i U_{l 3} m_{3}^{1 / 2} e^{-i \phi_{3} / 2}\right) \\
& \times\left(M_{1}^{*}\right)^{-1 / 2}, \quad R_{l N_{2}}= \pm i R_{l N_{1}}
\end{aligned}
$$

for normal hierarchy $(\mathrm{NH})$ of neutrino masses, and

$$
\begin{aligned}
R_{l N_{1}}= & \frac{1}{2} e^{\mp i x+|y|}\left(U_{l 1} m_{1}^{1 / 2} e^{-i \phi_{1} / 2} \mp i U_{l 2} m_{2}^{1 / 2} e^{-i \phi_{2} / 2}\right) \\
& \times\left(M_{1}^{*}\right)^{-1 / 2}, \quad R_{l N_{2}}= \pm i R_{l N_{1}}
\end{aligned}
$$

for inverted hierarchy ( $\mathrm{IH})$ of neutrino masses. $m_{1,2,3}$ are real masses of $\nu_{1,2,3}$, and $\phi_{1,2,3}$ the associated Majorana phases in diagonal form of $m_{\nu}$. For $\mathrm{NH}$, $m_{1}=0, m_{2}=\sqrt{\Delta m_{21}^{2}}, m_{3}=\sqrt{\left|\Delta m_{32}^{2}\right|+\Delta m_{21}^{2}}$. For IH, $m_{3}=0, m_{1}=\sqrt{\left|\Delta m_{32}^{2}\right|-\Delta m_{21}^{2}}, m_{2}=\sqrt{\left|\Delta m_{32}^{2}\right|} \cdot x$ and $y$ are two real free parameters to parametrize the mass matrix. Equations (6) and (7) are valid for large values of $y$, i.e., for the case that cancellation in (4) is needed to satisfy the $0 \nu \beta \beta$ constraint.

One can see in (6) and (7) that $\left|R_{l N_{2}}\right|^{2}=\left|R_{l N_{1}}\right|^{2}$ is valid for all flavors of neutrinos $\nu_{l=\tau, \mu, e}$, not just for $l=e$. This is one of the major properties of the low energy seesaw model if allowing large mixing of sterile neutrinos with active neutrinos. Using (6) and (7), one can also show the correlation of $\left|R_{l N_{1}}\right|^{2}$ by varying the free Dirac phase in matrix $U$ and the Majorana phases $\phi_{i}$. In Fig. 1 we plot the correlation of $\left(\left|R_{\tau N_{1}}\right|^{2}+\left|R_{\mu N_{1}}\right|^{2}\right) /\left|R_{e N_{1}}\right|^{2}$ versus $\left|R_{\tau N_{1}}\right|^{2} /\left|R_{\mu N_{1}}\right|^{2}$. In our computation we use [29]

$$
\begin{aligned}
& \sin ^{2} 2 \theta_{12}=0.846, \quad \sin ^{2} 2 \theta_{23}=0.999, \\
& \sin ^{2} 2 \theta_{13}=0.093,
\end{aligned}
$$

and

$$
\Delta m_{21}^{2}=7.53 \times 10^{-5} \mathrm{eV}^{2}, \quad\left|\Delta m_{32}^{2}\right|=2.48 \times 10^{-3} \mathrm{eV}^{2}
$$

For $\Delta m_{32}^{2}$ we have averaged two fit values for $\mathrm{NH}$ and $\mathrm{IH}$ [29]. One can see in these plots that the mixings of sterile neutrinos with $\nu_{\tau}$ and $\nu_{\mu}$ together are always stronger than the mixing with $\nu_{e}$ for $\mathrm{NH}$. For $\mathrm{IH},\left|R_{\tau N_{1}}\right|^{2}+\left|R_{\mu N_{1}}\right|^{2}$ can be larger than or smaller than $\left|R_{e N_{1}}\right|^{2}$. On the other hand, the ratio between $\left|R_{\tau N_{1}}\right|^{2}$ and $\left|R_{\mu N_{1}}\right|^{2}$ can be larger than or smaller than one for both $\mathrm{NH}$ and $\mathrm{IH}$.

From the above discussions, one can see that a major implication of a low energy seesaw type model with two $\mathrm{GeV}$ scale sterile neutrinos and large mixings with active neutrinos is the relation of mixings, such as $\left|R_{l N_{1}}\right|^{2}=$ $\left|R_{l N_{2}}\right|^{2}$ and the correlation shown in Fig. 1. For discussion of collider signatures in this low energy seesaw model, one should take these relations into account. However, as a first step towards this goal, we can discuss the signature of a single sterile neutrino with a mass at around $100 \mathrm{GeV}$. The signature of the low energy seesaw model can be obtained by extending the discussion for a single sterile neutrino to two sterile neutrinos and taking into account these relations among mixings described above. A further advantage of first discussing a single sterile neutrino is that the case of a single sterile neutrino may also be valid if other particle or mechanisms, e.g., some scalar particles and Type-II seesaw mechanism, are introduced. So a discussion on the collider signature of a single heavy sterile neutrino is of interest in and of itself. Needless to say, discussing the signature of a heavy sterile neutrino together with signature of other particles, e.g., scalar particles in type-II seesaw mechanism, is also of interest. In the present article, we are not going to elaborate on this topic. In the next section, we discuss the signature of a single sterile neutrino with a mass around $100 \mathrm{GeV}$ at CEPC. We come back to the signature of the low energy seesaw model in later sections. For previous works on the signature of heavy sterile neutrinos on $e^{+} e^{-}$ 
colliders, one can see a review in [24]. The present work gives a discussion on the signature of heavy sterile neutrino on CEPC within the framework of the low energy seesaw model and differs from the previous works in these aspect.

The mixings of sterile neutrinos with active neutrinos are also subject to the indirect constraints from tests of lepton universality, lepton flavor violation processes, and electroweak precision measurements [30-35]. For the heavy neutrino masses of order of $100 \mathrm{GeV},\left|R_{e N}\right|^{2},\left|R_{\mu N}\right|^{2}$, and $\left|R_{\tau N}\right|^{2}$ are constrained to be $\mathcal{O}\left(10^{-3}\right)$ mainly by the lepton flavor conserved decays of charged leptons, mesons, $W$, and $Z$. The combination $\left|R_{\mu N}^{*} R_{e N}\right|$ is stringently constrained to be order of $10^{-5}$ from the upper bounds of $\mu \rightarrow e \gamma$ and $\mu-e$ conversion. These indirect constraints are complementary to the probing of heavy sterile neutrinos at $e^{+} e^{-}$colliders. As will be shown in later sections, CEPC will probe $\left|R_{l N}\right|^{2}$ to $10^{-5}-10^{-6}$ and will have a better sensitivity than these indirect constraints at present.

\section{PRODUCTION AND DECAY OF A HEAVY STERILE NEUTRINO}

In this section, we discuss the production of a single heavy sterile neutrino at CEPC and its decay. CEPC under proposal plans to run electron-positron collision at a center of mass energy around $240 \mathrm{GeV}$ and aims at obtaining an integrated luminosity up to $5 \mathrm{ab}^{-1}$ with two interaction points and ten years of operation.

The Feynman diagrams of the production of a heavy Majorana-type sterile neutrino, $N$, are shown in Fig. 2. For simplicity, the heavy neutrino index $j$ will be suppressed in discussion for a single heavy sterile neutrino. The leading contribution to $N$ production is the process $e^{+} e^{-} \rightarrow N \nu_{l}\left(\bar{\nu}_{l}\right)$, the SM process $e^{+} e^{-} \rightarrow \nu_{l} \bar{\nu}_{l}$ with $\nu_{l}$ or $\bar{\nu}_{l}$ replaced by $N$ via its mixing with $\nu_{l}$. Because of the
Majorana nature of $\mathrm{N}$, it can mix with both $\nu_{l}$ and $\bar{\nu}_{l}$ with the same strength of mixing and can be produced via both of these mixings. These two possibilities are shown in the left and right panels in Fig. 2. As one can see in the upper panels of Fig. 2, the production of $N$ can be mediated by a $Z$ boson in s-channel with all types of neutrinos $\nu_{l}\left(\bar{\nu}_{l}\right)$ in final state. $N$ production can also be mediated by a $W$ boson in t-channel with $\nu_{e}\left(\bar{\nu}_{e}\right)$ in final state, as can be seen in the lower panels in Fig. 2. For the same strength of mixings, the t-channel process has a cross section two order of magnitude larger than the s-channel process and hence has a better sensitivities for the mixing $R_{e N}$.

We calculate the tree-level $e^{+} e^{-} \rightarrow N \nu_{l}$ cross sections with MadGraph [36] and implement the heavy neutrino interactions in FeynRules [37] with the Universal FeynRules Output (UFO) [38] format for the model. The results are shown in Fig. 3. For a heavy neutrino of about $100 \mathrm{GeV}$, the production cross section of $\sigma /\left|R_{e N}\right|^{2}$ and $\sigma /\left|R_{\mu N}\right|^{2}$ can reach $\sim 60 \mathrm{pb}$ and $\sim 0.8 \mathrm{pb}$ for only a single $R_{e N}$ mixing or $R_{\mu N}$ mixing, respectively.

Mixing of sterile neutrino $N$ with active neutrinos can lead to decay of $N$. For $m_{N}$, the mass of $N$, much smaller than $m_{W}$, the mass of a $W$ boson, the leading decays of $N$ are tree-level three-body decays mediated by off-shell $W$ or $Z$ bosons. Some three-body decay channels of $N$ are quite simple. For example, $N \rightarrow e^{-} \mu^{+} \nu_{\mu}$ is mediated by an off-shell $W$ boson and is similar to $\mu \rightarrow \nu_{\mu} e \bar{\nu}_{e}$, the leptonic decay of $\mu$, except with the presence of a mixing factor $\left|R_{e N}\right|^{2}$ in decay rate. Some decay channels, e.g., $N \rightarrow \nu_{e} e^{-} e^{+}$, can be mediated by both off-shell $W$ and $Z$ bosons. But it does not introduce complications in the decay rate. The results are presented in (A18)-(A24) in the Appendix.

For $m_{N}$ much greater than $m_{W}$ and $m_{Z}$, the leading decay of $N$ are two-body decays, $N \rightarrow l^{ \pm} W^{\mp}$ and $N \rightarrow \nu(\bar{\nu}) Z$.

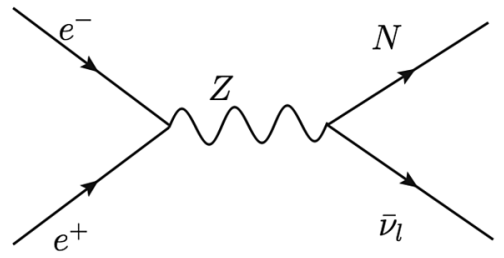

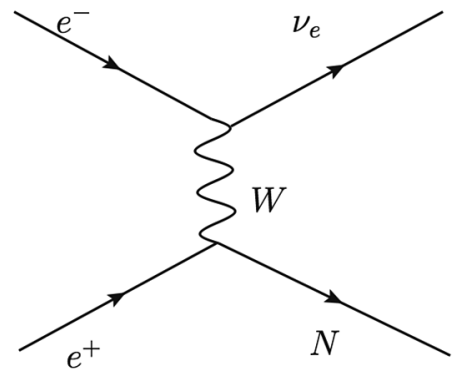

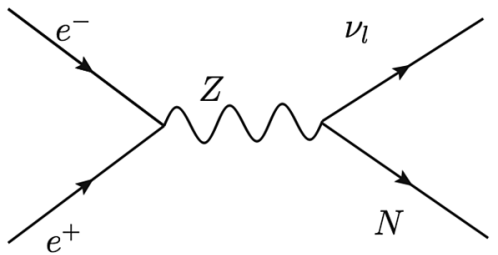

FIG. 2. Feynman diagrams. 

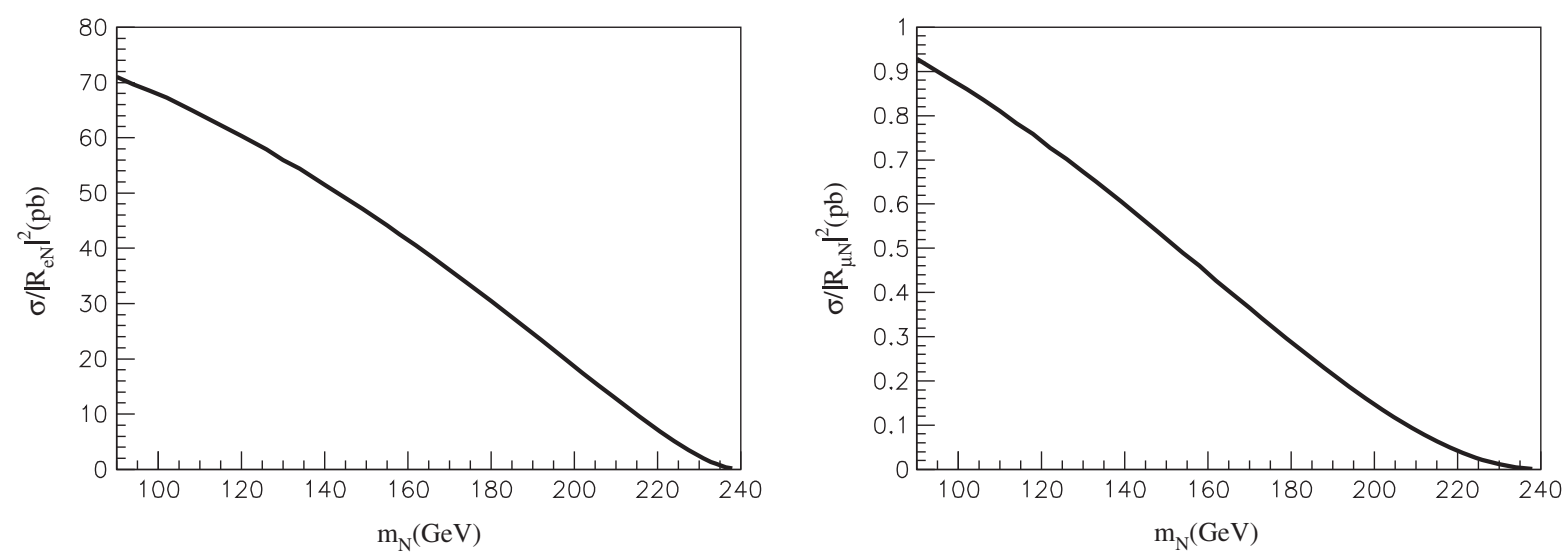

FIG. 3. $e^{+} e^{-} \rightarrow N \nu$ cross section at $\sqrt{s}=240 \mathrm{GeV}$ with only a single $R_{e N}$ mixing (left) and $R_{\mu N}$ mixing (right).

For $m_{N}$ greater than $m_{H}$, the mass of the Higgs boson, $N$ can also decay to $H$ via $N \rightarrow \nu(\bar{\nu}) H$. The partial decay widths of the heavy neutrino can be written as $[3,21,39,40]$

$\Gamma\left(N \rightarrow l^{-} W^{+}\right)=\frac{g^{2}}{64 \pi}\left|R_{l N}\right|^{2} \frac{m_{N}^{3}}{m_{W}^{2}}\left(1-\mu_{W}\right)^{2}\left(1+2 \mu_{W}\right)$

$\Gamma(N \rightarrow \nu Z)=\frac{g^{2}}{64 \pi}\left|R_{l N}\right|^{2} \frac{m_{N}^{3}}{m_{W}^{2}}\left(1-\mu_{Z}\right)^{2}\left(1+2 \mu_{Z}\right)$

$\Gamma(N \rightarrow \nu H)=\frac{g^{2}}{64 \pi}\left|R_{l N}\right|^{2} \frac{m_{N}^{3}}{m_{W}^{2}}\left(1-\mu_{H}\right)^{2}$

with $\mu_{i}=m_{i}^{2} / m_{N}^{2}(i=W, Z, H)$. W, Z, or $H$ eventually decay to fermions. Hence, the decay rate to a specific threebody final state can be calculated using (10)-(12) and the branching ratio of $W, Z$, or $H$ to a specific fermion pair. For example, $\Gamma\left(N \rightarrow e^{-} \mu^{+} \nu_{\mu}\right)$ is obtained using $\Gamma\left(N \rightarrow e^{-} W^{+}\right)$ and $\operatorname{Br}\left(W \rightarrow \mu^{+} \nu_{\mu}\right)$ as $\Gamma\left(N \rightarrow e^{-} \mu^{+} \nu_{\mu}\right)=\Gamma\left(N \rightarrow e^{-} W^{+}\right) \times$ $\operatorname{Br}\left(W^{+} \rightarrow \mu^{+} \nu_{\mu}\right)$, where $\operatorname{Br}\left(W^{+} \rightarrow \mu^{+} \nu_{\mu}\right)$ is the branching ratio of $W^{+} \rightarrow \mu^{+} \nu_{\mu}$ decay.

For more general values of $m_{N}$, in particular for $m_{N} \approx m_{W, Z, H}$, the above formulas are not good approximations. Decay rate in more general cases can be calculated by carefully including the propagators of $W, Z$, and $H$ bosons into calculation. The four-momentum of the mediated boson can be on-shell for general cases. We take this fact into account and calculate the tree-level decay rate of $N$ decays with three fermions in final state. In the Appendix, we present in detail the results of our calculations. One can see that for most cases the decay rate can be obtained as an analytic function of $m_{N}$ and the masses and widths of bosons. The most complicated case appears for $N \rightarrow l^{-} l^{+} \nu_{l}$ and $N \rightarrow l^{-} l^{+} \bar{\nu}_{l}$ channels for which $W$ and $Z$ bosons can all mediate. For this particular process, a function $F_{S}$, shown in (A5) and (A15), appears, which cannot be obtained as an explicit analytic function of $m_{N}$ and the boson masses. In our analysis we compute $F_{S}$ numerically.

As an example, we compare in Fig. 4 the result computed using an analytic formula (A3) with known results in the low energy region $m_{N} \ll m_{W}$ and in the high energy limit $m_{N}>m_{W} . \quad \Gamma\left(N \rightarrow e^{-} W^{+}\right)$is calculated using (10). $\operatorname{Br}\left(W^{+} \rightarrow \mu^{+} \nu_{\mu}\right)$ is taken as $\mathrm{Br}=0.108$ [29]. We can see that in the low energy limit the decay rate agrees with the expected result of tree-level three-body decay. In the high energy limit it agrees with the expectation that it is dominated by the on-shell $N \rightarrow e^{-} W^{+}$decay with a subsequent $W^{+} \rightarrow \mu^{+} \nu_{\mu}$ decay. In the region around $m_{W}$, (A3) gives a smooth transition from low energy behavior to high energy behavior. As a comparison, the result calculated using the two body decay $N \rightarrow e^{-} W^{+}$drops down to zero as $m_{N}$ approaches $m_{W}$ from above and is certainly not correct at around the threshold. The result given by (A3) takes into account the contribution of the off-shell boson and removes the ill behavior at around $m_{N} \sim m_{W}$. The plot demonstrates that the results presented in the Appendix are

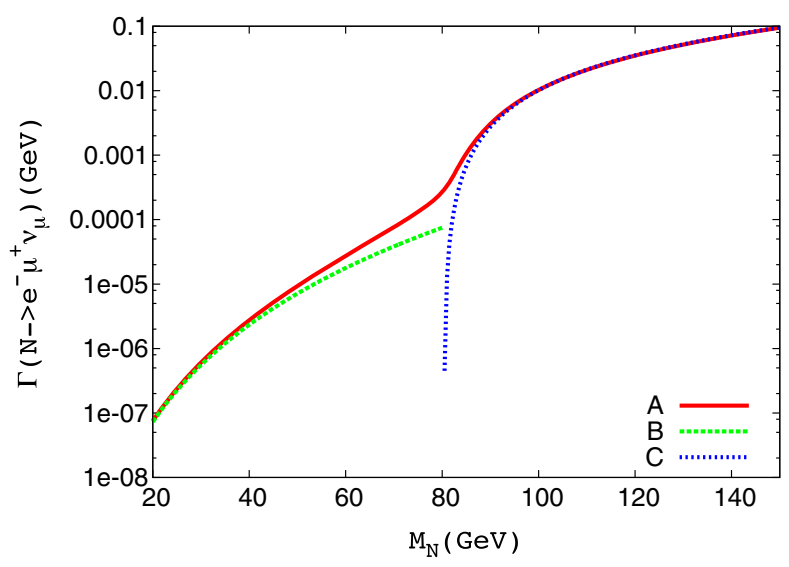

FIG. 4. Decay rate of $N \rightarrow e^{-} \mu^{+} \nu_{\mu}$ versus $m_{N}$ with $\left|R_{e N}\right|^{2}=1$. Line A: calculated using (A3); Line B: calculated using $\Gamma=$ $G_{F}^{2} m_{N}^{5} /\left(192 \pi^{3}\right)$ in (A18) up to $m_{N}<m_{W}$; Line C: calculated using $\Gamma=\Gamma\left(N \rightarrow e^{-} W^{+}\right) \operatorname{Br}\left(W^{+} \rightarrow \mu^{+} \nu_{\mu}\right)$ with $m_{N}>m_{W}$. 
better to use for studying the signals of the sterile neutrino. Tree-level three-body decay rates for general mass $m_{N}$, presented in the Appendix, are some of the new results of the present article.

\section{SIGNAL OF A HEAVY STERILE NEUTRINO AND BACKGROUND}

In this section, we study the process

$$
e^{+} e^{-} \rightarrow N \nu, \quad N \bar{\nu} \rightarrow l j j \not E,
$$

the signal of sterile neutrino $N$ due to this process, and the associated background.

We simulate the signal and background events with MadGraph [36], and have done the showing and hadronization by using Pythia6 [41]. The results are passed through PGS4 [42] for fast detector simulation.

At CEPC with $\sqrt{s}=240 \mathrm{GeV}$, we adopt the basic cuts (BC) for lepton and jets to select the events,

$$
\begin{aligned}
& p_{T}^{l}>10 \mathrm{GeV}, \quad\left|\eta^{l}\right|<2.5, \quad \Delta R_{l l}>0.4, \\
& p_{T}^{j}>10 \mathrm{GeV}, \quad\left|\eta^{j}\right|<2.5, \quad \Delta R_{j j}>0.4, \quad \Delta R_{l j}>0.4 .
\end{aligned}
$$

The main backgrounds for the process (13) are $W$ pair production, $e^{+} e^{-} \rightarrow W^{+} W^{-}$, with one $W$ decaying leptonically and the other $W$ decaying hadronically, and single $W$ production, which decays leptonically. In order to suppress the backgrounds, we set the selection cuts (SC) [21,39],

$$
\left|M(l, \mathbb{E})-m_{W}\right|>20 \mathrm{GeV},
$$

and

$$
\left|M\left(l, j_{1}, j_{2}\right)-m_{N}\right|<20 \text { or } 10 \mathrm{GeV}
$$

Cut (16) is used to exclude background events coming from the decay of on-shell $W$ boson in the background processes. Cut (17) selects events coming from the decay of on-shell $N$ and is used to increase the significance of signal-tobackground ratio.

In Table I we show the efficiency of the cuts for both $l=e$ and $l=\mu$ channels. After adding the SC, the signals are survived, but the backgrounds drop several order of magnitude.

We define the significance $s$ as

$$
s=\frac{\mathcal{N}_{s}}{\sqrt{\mathcal{N}_{s}+\mathcal{N}_{b}}},
$$

where $\mathcal{N}_{s}$ and $\mathcal{N}_{b}$ are the event number of signal and background respectively. In Fig. 5 we plot the significance $s$ versus $m_{N}$ for $l=e$ with $R_{e N}=0.015$ and $l=\mu$ with $R_{\mu N}=0.1$, respectively. For the integrated luminosity of $100 \mathrm{fb}^{-1}$, a heavy neutral neutrino with mass in the range of $90 \mathrm{GeV} \leq m_{N} \leq 146 \mathrm{GeV}$ for the mixing $R_{e N}=0.015$ is promised to be discovered in the $l=e$ channel, and $90 \mathrm{GeV} \leq m_{N} \leq 150 \mathrm{GeV}$ for the mixing $R_{\mu N}=0.1$ is promised to be discovered in the $l=\mu$ channel. For the integrated luminosity of $5 \mathrm{ab}^{-1}$, the maximal values of heavy neutrino mass can be $235 \mathrm{GeV}$ and $205 \mathrm{GeV}$ for the $l=e$ and $l=\mu$ channel, respectively. One can see that there is a quick drop for heavy neutrinos with mass $\lesssim 100 \mathrm{GeV}$ for both of $l=e$ and $l=\mu$. This is because for the decay of $N$ of a mass $\lesssim 100 \mathrm{GeV}$, the lepton in $N \rightarrow l W \rightarrow l j j$, a decay chain with an almost on-shell $W$, does not have enough energy and the $p_{T}$ of $l$ cannot be large. This effect of cut on $p_{t}$ of $l$ can be seen in Table I C. In Fig. 5, one can also see that there is a small peak for a heavy neutrino with mass around $230 \mathrm{GeV}$. This is because of the cut $\left|M(l, E)-m_{W}\right|>20 \mathrm{GeV}$ to the signal as shown in Table I E. Compared with the case of $m_{N}=214 \mathrm{GeV}$ in

\begin{tabular}{|c|c|c|c|c|c|c|c|}
\hline & parameters & +cuts (a) & + cuts (b) & + cuts $(\mathrm{c})$ & + cuts (d) & + cuts (e) & significance \\
\hline \multirow[t]{2}{*}{$\mathrm{A}$} & $m_{N}=150 \mathrm{GeV}$ & 2.14 & 2.04 & 1.56 & 1.56 & 1.55 & 11.2 \\
\hline & $R_{\mu N}=0.1$ & $2.31 \times 10^{3}$ & $2.20 \times 10^{3}$ & 52.4 & 16.3 & 8.05 & \\
\hline \multirow[t]{2}{*}{ B } & $m_{N}=150 \mathrm{GeV}$ & 7.63 & 7.30 & 5.61 & 5.60 & 5.60 & 18.8 \\
\hline & $R_{e N}=0.02$ & $2.52 \times 10^{3}$ & $2.37 \times 10^{3}$ & $0.195 \times 10^{3}$ & 76.6 & 38.8 & \\
\hline \multirow[t]{2}{*}{$\mathrm{C}$} & $m_{N}=90 \mathrm{GeV}$ & 10.8 & 4.98 & 1.56 & 1.55 & 1.55 & 13.4 \\
\hline & $R_{e N}=0.015$ & $2.52 \times 10^{3}$ & $2.37 \times 10^{3}$ & $0.195 \times 10^{3}$ & 16.8 & 5.14 & \\
\hline \multirow[t]{2}{*}{$\mathrm{D}$} & $m_{N}=214 \mathrm{GeV}$ & 0.852 & 0.827 & 0.243 & 0.242 & 0.241 & 1.75 \\
\hline & $R_{e N}=0.015$ & $2.52 \times 10^{3}$ & $2.37 \times 10^{3}$ & $0.195 \times 10^{3}$ & 24.9 & 9.26 & \\
\hline \multirow[t]{2}{*}{$\mathrm{E}$} & $m_{N}=230 \mathrm{GeV}$ & 0.194 & 0.188 & 0.160 & 0.160 & 0.160 & 2.76 \\
\hline & $R_{e N}=0.015$ & $2.54 \times 10^{3}$ & $2.39 \times 10^{3}$ & $0.197 \times 10^{3}$ & 4.14 & 1.49 & \\
\hline
\end{tabular}
Table I D, a heavier neutrino with mass of $230 \mathrm{GeV}$ tends to move more slowly in the center of mass system of colliding

TABLE I. The cross sections (unit fb) of signal (upper line) after imposing various cuts (a, b, c, d, e) sequentially, the background (lower line) and the significance after cuts with integrated luminosity of $500 \mathrm{fb}^{-1}$. Cuts (a) $p_{T}^{j, l}>1 \mathrm{GeV}$, (b) $p_{T}^{j, l}>10 \mathrm{GeV}$, (c) $\left|M(l, \mathbb{E})-m_{W}\right|>20 \mathrm{GeV}$, (d) $\left|M\left(l, j_{1}, j_{2}\right)-m_{N}\right|<20 \mathrm{GeV}$, (e) $\left|M\left(l, j_{1}, j_{2}\right)-m_{N}\right|<10 \mathrm{GeV}$. 

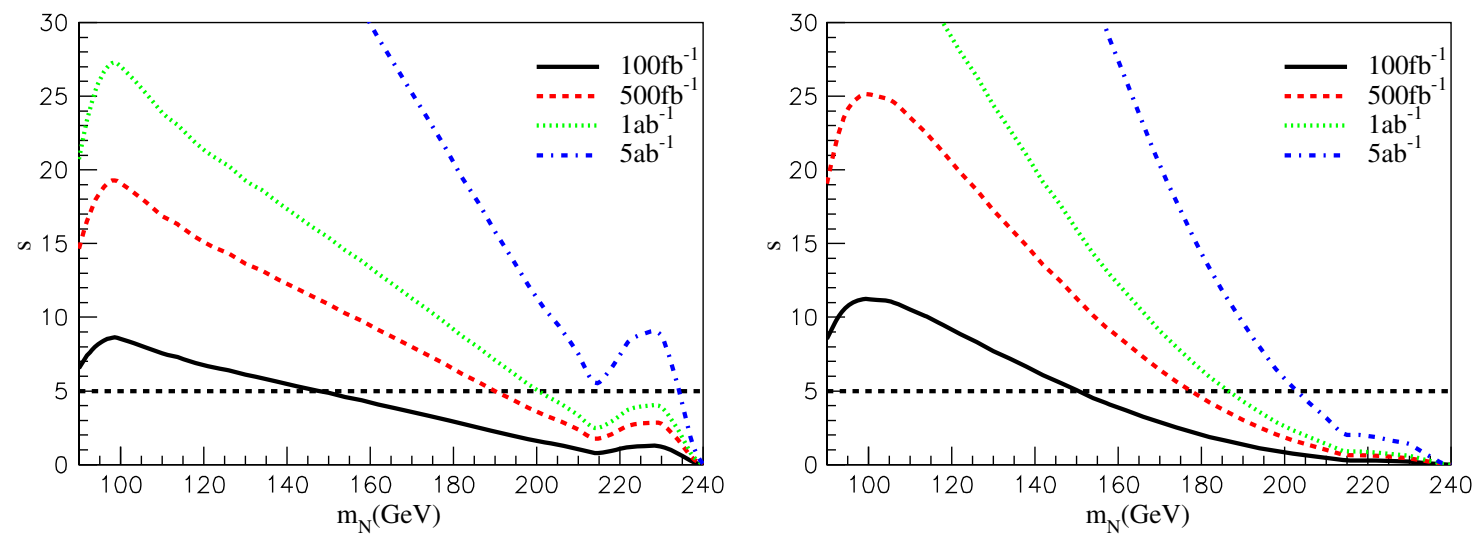

FIG. 5. The significance for $l=e$ (left) with $R_{e N}=0.015$ and $l=\mu$ (right) with $R_{\mu N}=0.1$. The curves in each plot from up to down correspond to the integrated luminosities $5 \mathrm{ab}^{-1}, 1 \mathrm{ab}^{-1}, 500 \mathrm{fb}^{-1}$, and $100 \mathrm{fb}^{-1}$.

$e^{+} e^{-}$, and it decays to a charged lepton which distributes more uniformly in all directions. More importantly, the light neutrino, produced together with the heavier sterile neutrino with a mass of $230 \mathrm{GeV}$, becomes quite soft (with an energy $\approx 9.8 \mathrm{GeV}$ ). Then, the invariant mass of the light neutrino and charged lepton $M(l, \mathbb{E})$ will distribute more evenly. Consequently, the cut $\left|M(l, \not{E})-m_{W}\right|>20 \mathrm{GeV}$ does not hurt the signal as much as in the case of
$m_{N}=214 \mathrm{GeV}$, as shown in Table I D and E. This can be verified if the cut $(c)$ is changed to $\left|M(l, \mathbb{E})-m_{W}\right|>$ $10(30) \mathrm{GeV}$, the signal cross section is changed to 0.188 $(0.103) \mathrm{fb}$, respectively. On the other hand, the background cross section reduces significantly with $m_{N}=230 \mathrm{GeV}$ in Table I E after adding all the cuts.

In Fig. 6 we plot the potential of probing $R_{l N}$ for a fixed significance $s=5$ with the integrated luminosities $5 \mathrm{ab}^{-1}$,
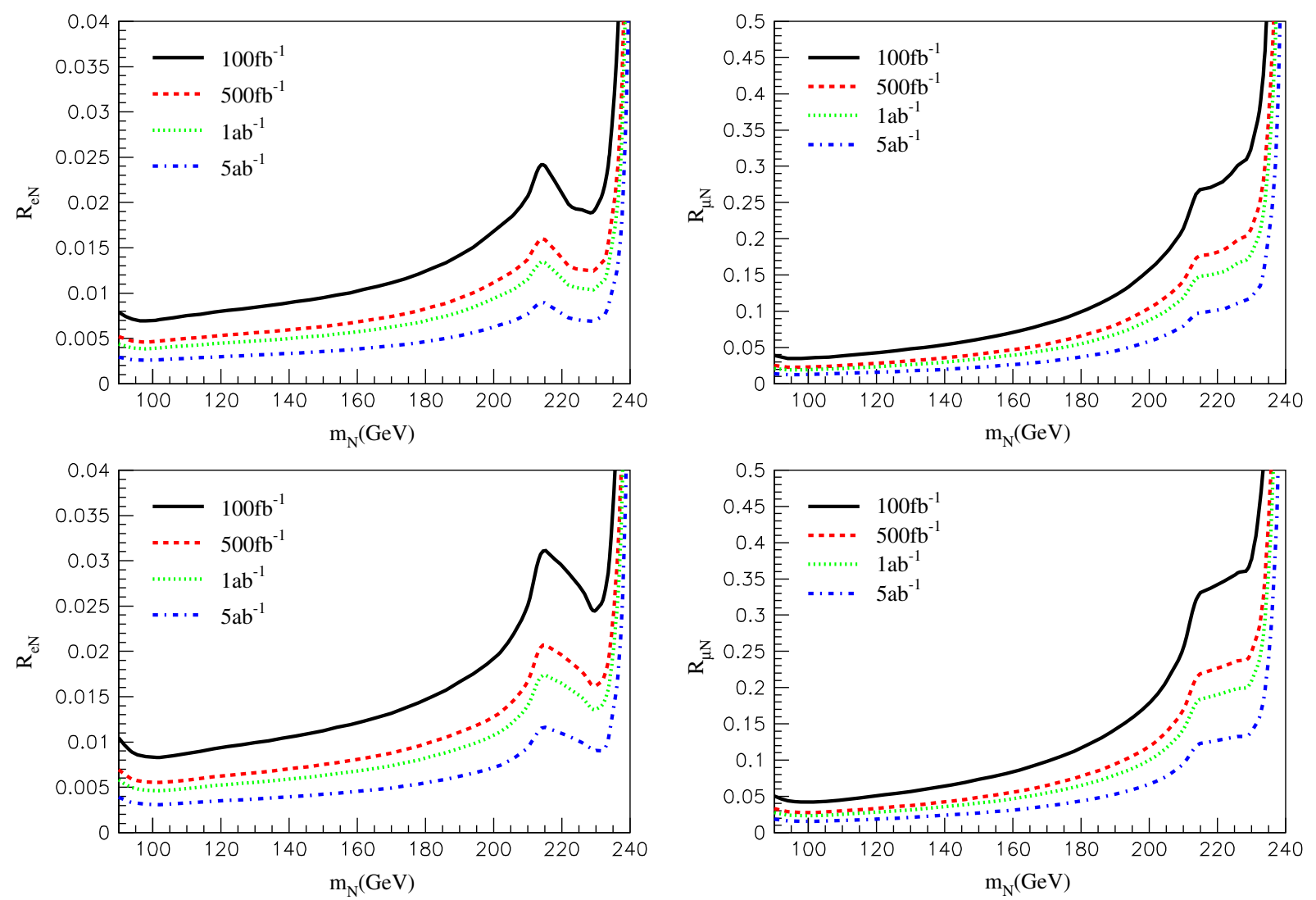

FIG. 6. Sensitivity to $R_{l N}(l=e, \mu)$ with significance $s=5$. The upper plots are for $\mathrm{SC}\left|M\left(l, j_{1}, j_{2}\right)-m_{N}\right|<10 \mathrm{GeV}$ and the lower plots are for SC $\left|M\left(l, j_{1}, j_{2}\right)-m_{N}\right|<20 \mathrm{GeV}$. The curves in each plot from up to down correspond to integrated luminosities $100 \mathrm{fb}^{-1}$, $500 \mathrm{fb}^{-1}, 1 \mathrm{ab}^{-1}$, and $5 \mathrm{ab}^{-1}$. 
$1 \mathrm{ab}^{-1}, 500 \mathrm{fb}^{-1}$, and $100 \mathrm{fb}^{-1}$ at CEPC. Using SC $\left|M\left(l, j_{1}, j_{2}\right)-m_{N}\right|<10 \mathrm{GeV}$, in the $l=e$ channel, a heavy neutrino mass of $120 \mathrm{GeV}$ with $R_{e N}=0.0080$ can be discovered for the integrated luminosities $100 \mathrm{fb}^{-1}$, and for $5 \mathrm{ab}^{-1}$, the mixing as low as $R_{e N}=$ 0.0030 for the same mass can be probed. In the $l=\mu$ channel, the heavy neutrino of the same mass with $R_{\mu N}=$ 0.043 can be discovered for $100 \mathrm{fb}^{-1}$, and $R_{\mu N}=0.016$ for $5 \mathrm{ab}^{-1}$. We can have similar results for SC $\mid M\left(l, j_{1}, j_{2}\right)-$ $m_{N} \mid<20 \mathrm{GeV}$, but the corresponding mixings are a little bigger.

\section{SIGNAL OF LOW ENERGY SEESAW MODEL}

In this section we discuss the signature of the low energy seesaw model with two heavy sterile neutrinos of mass around $100 \mathrm{GeV}$.

As discussed in previous section, in the case of large mixing of heavy sterile neutrinos with active neutrinos, not only the masses of these two sterile neutrinos are (quasi) degenerate but also the mixing has a simple relation $R_{l N_{2}}=$ $\pm i R_{l N_{1}}$ as shown in (6) and (7). So the signature of the low energy seesaw model discussed here is just the double of the result presented for a single heavy sterile neutrino, except that we need to take into account the correlation of the mixing $R_{l N}$ for different $l$ in the low energy seesaw model, as shown in Fig. 1.

We calculate the signal of $e^{+} e^{-} \rightarrow \nu l j j$ events and the related background for $l=e, \mu, \tau$ separately. Then we calculate the significance of $e^{+} e^{-} \rightarrow \nu l j j$ events for $l=e$, $\mu, \tau$ separately. The total significance is defined as the square root of the sum of the squares of the significances of signals of $l=e, l=\mu$, and $l=\tau$, which we call $e+\mu+\tau$ significance. Similarly, we can define $e+\mu$ significance, which includes signals of $l=e$ and $l=\mu$. For simplicity, we assume $100 \%$ efficiency of the identification of the $\tau$ lepton. A realistic efficiency can be put into analysis

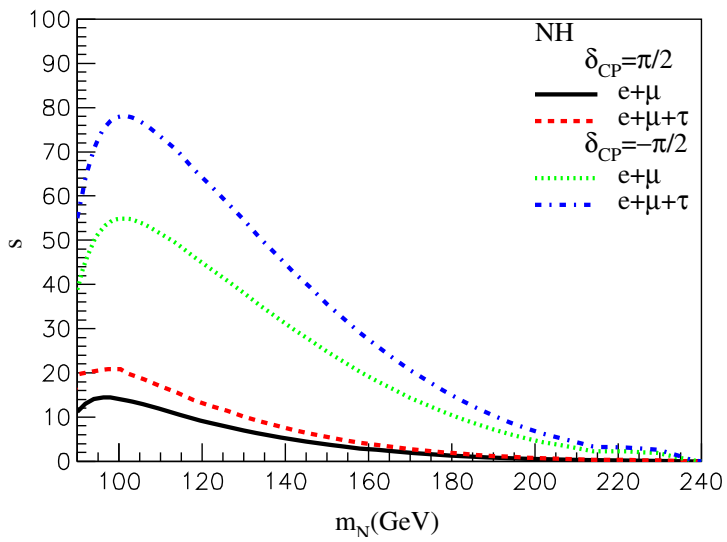

without difficulty and would give rise to a result between the lines of $e+\mu+\tau$ significance and $e+\mu$ significance presented in figures below.

We plot the significance versus the mass of heavy neutrinos in Fig. 7 for $\mathrm{NH}$ and $\mathrm{IH}$ with parameters given in the caption and with integrated luminosity $500 \mathrm{fb}^{-1}$ as an illustration. In the case of $\mathrm{NH}$, we choose to have $\left|R_{\mu N}\right|$ about 10 times larger than $\left|R_{e N}\right|$ for $\delta_{\mathrm{CP}}=\pi / 2 . R_{\tau N}$ is of the same magnitude as $R_{\mu N}$, so the dominant decay channels are the $\mu$ and $\tau$ channels which dominate the total significance in the figure. For $\delta_{\mathrm{CP}}=-\pi / 2,\left|R_{\mu N}\right|$ is of the same size of $\left|R_{\tau N}\right|$, but approximately 2 times larger than $\left|R_{e N}\right|$. Furthermore, the backgrounds for the $\mu$ and $\tau$ channels are several times smaller than the $e$ channel. Thus, the $\mu$ and $\tau$ channels are still dominant in $e+\mu+\tau$ significance.

As can be seen in Fig. 7, in the case of NH, a heavy sterile neutrino with a mass less than about $152 \mathrm{GeV}$ $\left(\left|R_{e N}\right| \sim 0.0032,\left|R_{\mu N}\right| \sim\left|R_{\tau N}\right| \sim 0.034\right)$ can be discovered for $\delta_{\mathrm{CP}}=\pi / 2$. For $\delta_{\mathrm{CP}}=-\pi / 2$, a heavy sterile neutrino with a mass less than around $206 \mathrm{GeV}\left(\left|R_{e N}\right| \sim 0.015\right.$, $\left.\left|R_{\mu N}\right| \sim\left|R_{\tau N}\right| \sim 0.028\right)$ can be discovered. As can be seen in the above example, the case with $\delta_{\mathrm{CP}}=-\pi / 2$ has a larger $\left|R_{e N}\right|$. This larger value of $\left|R_{e N}\right|$ enhances the t-channel production process and gives rise to a larger production rate of heavy sterile neutrinos. Meanwhile, the $\mu$ or $\tau$ channel decay of $N$ is still dominating over the $e$ channel, so the significance increases a lot from the case of $\delta_{\mathrm{CP}}=\pi / 2$ to the case of $\delta_{\mathrm{CP}}=-\pi / 2$.

In the case of IH, we choose to have similar magnitude of $\left|R_{e N}\right|,\left|R_{\mu N}\right|$, and $\left|R_{\tau N}\right|$ for both cases of $\delta_{\mathrm{CP}}=\pi / 2$ and $\delta_{\mathrm{CP}}=-\pi / 2$. All three $e, \mu$, and $\tau$ decay channels have comparable contributions to the total significance. For the Dirac phase of both cases of $\delta_{\mathrm{CP}}=\pi / 2$ and $\delta_{\mathrm{CP}}=-\pi / 2$, the magnitude of $\left|R_{e N}\right|$ has the same size, and so does $\left|R_{e N}\right|^{2}+\left|R_{\mu N}\right|^{2}+\left|R_{\tau N}\right|^{2}$. This leads to the same production rate of $e^{+} e^{-} \rightarrow \nu N$ and the same $l j j$ decays of $N$ for both

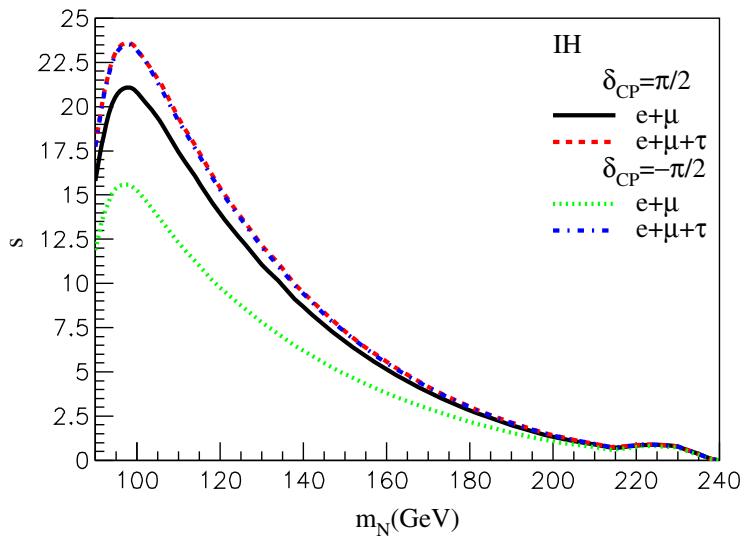

FIG. 7. The significance $s$ vs $m_{N}$ for NH (left) and IH (right) with integrated luminosity $500 \mathrm{fb}^{-1}$. We choose $e^{y}=5000, \delta_{\mathrm{CP}}= \pm \pi / 2$, $\phi_{1}=\phi_{2}=\phi_{3}=0$ for NH (the largest eigenvalue of the matrix of neutrino Yukawa couplings defined in Ref. [32] is $0.00495 \times \sqrt{m_{N}}$ ), and $e^{y}=1000, \delta_{\mathrm{CP}}= \pm \pi / 2, \phi_{1}=\phi_{2}=\phi_{3}=0$ for IH (the largest eigenvalue of the matrix of neutrino Yukawa couplings is $\left.0.00128 \times \sqrt{m_{N}}\right)$. 

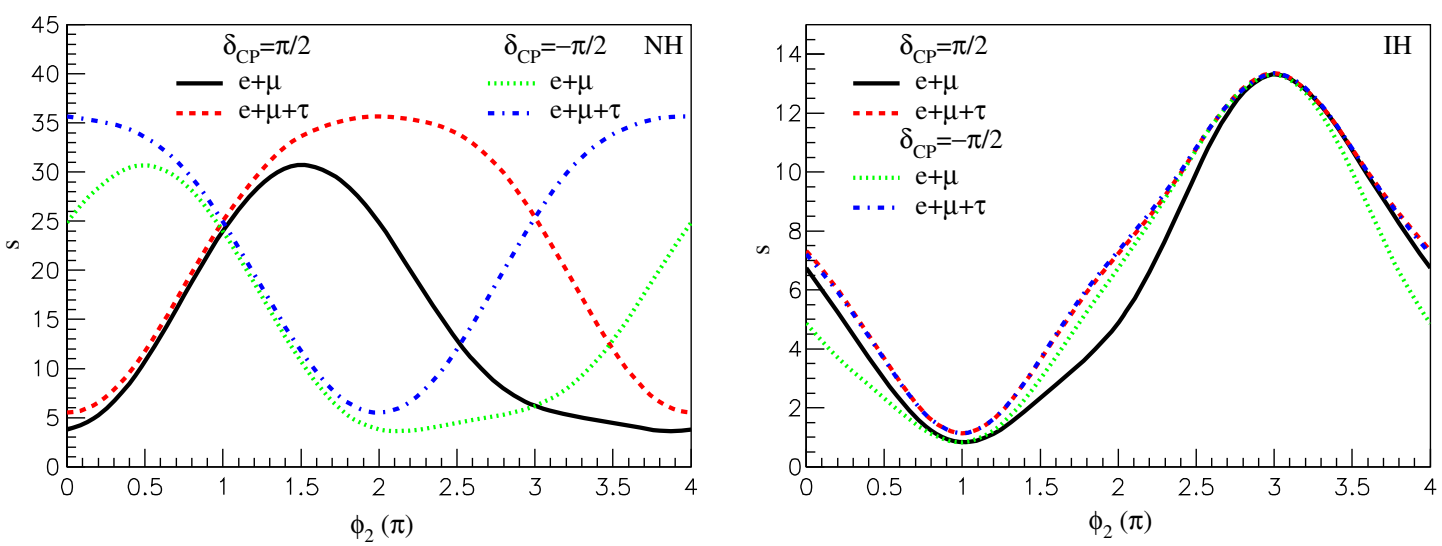

FIG. 8. The significance $s$ vs $\phi_{2}$ for $\mathrm{NH}$ (left) and IH (right) with integrated luminosity $500 \mathrm{fb}^{-1}$ for a heavy neutrino mass of $150 \mathrm{GeV}$. We choose $e^{y}=5000, \delta_{\mathrm{CP}}= \pm \pi / 2, \phi_{1}=\phi_{3}=0$ for $\mathrm{NH}$ (the largest eigenvalue of the matrix of neutrino Yukawa couplings is 0.0606 ), and $e^{y}=1000, \delta_{\mathrm{CP}}= \pm \pi / 2, \phi_{1}=\phi_{3}=0$ for $\mathrm{IH}$ (the largest eigenvalue of the matrix of neutrino Yukawa couplings is 0.0156 ).

cases of $\delta_{\mathrm{CP}}=\pi / 2$ and $\delta_{\mathrm{CP}}=-\pi / 2$. So, the total $e+\mu+\tau$ significances are the same for both cases of $\delta_{\mathrm{CP}}=\pi / 2$ and $\delta_{\mathrm{CP}}=-\pi / 2$. However, there is a difference between the $e+\mu$ significances for these two cases. One can see in Fig. 7 that for $\mathrm{IH}$ a heavy neutrino with mass less than about $162 \mathrm{GeV}$ can be discovered. The corresponding mixing parameters in the figure are $\left|R_{e N}\right| \sim 0.0086,\left|R_{\mu N}\right| \sim$ $0.0072,\left|R_{\tau N}\right| \sim 0.0051$ for $\delta_{\mathrm{CP}}=\pi / 2$, and $\left|R_{e N}\right| \sim 0.0086$, $\left|R_{\mu N}\right| \sim 0.0053,\left|R_{\tau N}\right| \sim 0.0071$ for $\delta_{\mathrm{CP}}=-\pi / 2$.

In Fig. 8, we also plot the total significance as a function of the Majorana phase $\phi_{2}$ for a heavy neutrino mass of $150 \mathrm{GeV}$ and integrated luminosity of $500 \mathrm{fb}^{-1}$ for both $\mathrm{NH}$ and $\mathrm{IH}$. The significance depends on both the Dirac phase of $\delta_{\mathrm{CP}}$ and Majorana phase $\phi_{2}$. In the case of $\mathrm{NH}$ with $\delta_{\mathrm{CP}}=\pi / 2$, there is a bump at $\phi_{2} \sim 1.5 \pi$ for $e+\mu$ significance. On the other hand, the bump is at around $2 \pi$ for $e+\mu+\tau$ significance. This is because $\left|R_{e N}\right|^{2} / \sum\left|R_{l N}\right|^{2}$ increases as $\phi_{2}$ increases from 0 to $2 \pi$, as can be seen in Fig. 9. Since $\sum\left|R_{l N}\right|^{2}$ is a constant when varying $\phi_{2}$, as can

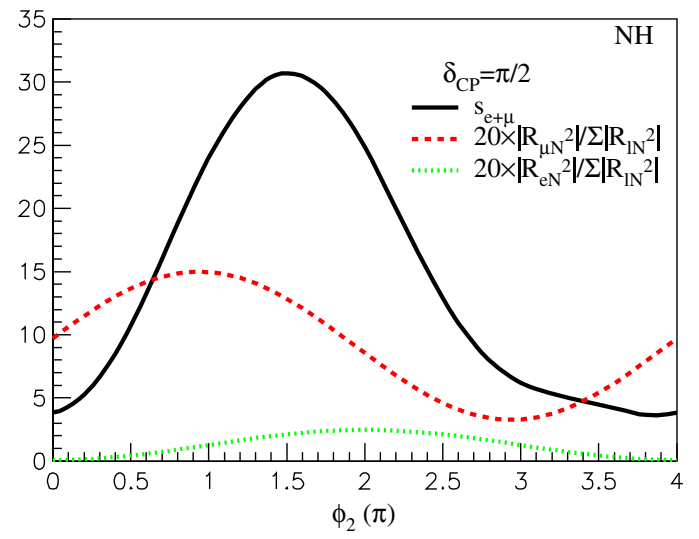

FIG. 9. The significance $s$ vs $\phi_{2}$ for $\mathrm{NH}$ with $\delta_{\mathrm{CP}}=\pi / 2$ with $e^{y}=5000, \phi_{1}=\phi_{3}=0$ (the largest eigenvalue of the matrix of neutrino Yukawa couplings is 0.0606 ), and integrated luminosity $500 \mathrm{fb}^{-1}$. be easily checked using (6) and (7), $\left|R_{e N}\right|^{2}$ increases as $\phi_{2}$ increases from 0 to $2 \pi$ and peaks at $\phi_{2}=2 \pi$. Consequently, the $\mathrm{t}$-channel production process, the dominating production process, increases as $\phi_{2}$ increases from 0 to $2 \pi$. This is why the plot of the $e+\mu+\tau$ significance peaks at $\phi_{2}=2 \pi$ in the case of $\mathrm{NH}$ with $\delta_{\mathrm{CP}}=\pi / 2$. For the $e+\mu$ significance, it is dominated by the $\mu j j$ events, as explained before. As $\phi_{2}$ increases, $\left|R_{\mu N}\right|^{2} / \sum\left|R_{l N}\right|^{2}$ peaks at $\phi_{2} \sim \pi$. For $\phi_{2}$ larger than around $\pi$, the branching fraction of the $N \rightarrow \mu j j$ decay starts to decrease, which is compensated by the increase of the production cross section of $e^{+} e^{-} \rightarrow N \nu$. Then, the signature of $\mu j j$ events will increase first and then decrease as $\phi_{2}$ increases from $\pi$ to $2 \pi$. This makes $e+\mu$ significance having a peak at a position less than $2 \pi$, as can seen in Fig. 8. Variation of significance in other cases can be similarly understood.

In Fig. 10, we present the significance as a function of heavy neutrino mass with integrated luminosity $5 \mathrm{ab}^{-1}$. In the case of $\mathrm{NH}$, a heavy neutrino mass less than about $124 \mathrm{GeV}\left(\left|R_{e N}\right| \sim 0.0012,\left|R_{\mu N}\right| \sim\left|R_{\tau N}\right| \sim 0.013\right)$ for $\delta_{\mathrm{CP}}=$ $\pi / 2$, and $184 \mathrm{GeV}\left(\left|R_{e N}\right| \sim 0.0055,\left|R_{\mu N}\right| \sim\left|R_{\tau N}\right| \sim 0.010\right)$ for $\delta_{\mathrm{CP}}=-\pi / 2$ can be discovered at CEPC. In the case of $\mathrm{IH}$, a heavy neutrino mass less than about $130 \mathrm{GeV}$ can be discovered. The corresponding mixing parameters are $\left|R_{e N}\right| \sim 0.0034,\left|R_{\mu N}\right| \sim 0.0028,\left|R_{\tau N}\right| \sim 0.0020$ for $\delta_{\mathrm{CP}}=$ $\pi / 2$, and $\left|R_{e N}\right| \sim 0.0034,\left|R_{\mu N}\right| \sim 0.0021,\left|R_{\tau N}\right| \sim 0.0028$ for $\delta_{\mathrm{CP}}=-\pi / 2$.

In Fig. 11, we plot the potential of probing $\left|R_{\mu N}\right|$ for $e+\mu$ (or $e+\mu+\tau$ ) significance $s=5$ with the integrated luminosities $5 \mathrm{ab}^{-1}, 1 \mathrm{ab}^{-1}, 500 \mathrm{fb}^{-1}$ and $100 \mathrm{fb}^{-1}$ at CEPC for different cases of $\mathrm{NH}$ and $\mathrm{IH}$, and Dirac phase $\delta_{\mathrm{CP}}= \pm \pi / 2$. For each case, the ratio of $\left|R_{e N}\right|:\left|R_{\mu N}\right|:\left|R_{\tau N}\right|$ is fixed for the given Dirac phase and Majorana phases; therefore we only plot $\left|R_{\mu N}\right|$ for illustration. In the case of $\mathrm{NH}$, the ratio of $\left|R_{e N}\right|:\left|R_{\mu N}\right|:\left|R_{\tau N}\right|$ is $0.0945: 1: 1.03$ $(0.537: 1: 1.02)$ for $\delta_{\mathrm{CP}}=\pi / 2\left(\delta_{\mathrm{CP}}=-\pi / 2\right)$. In the case of $\mathrm{IH}$, the ratio of $\left|R_{e N}\right|:\left|R_{\mu N}\right|:\left|R_{\tau N}\right|$ is 1.19:1:0.709 

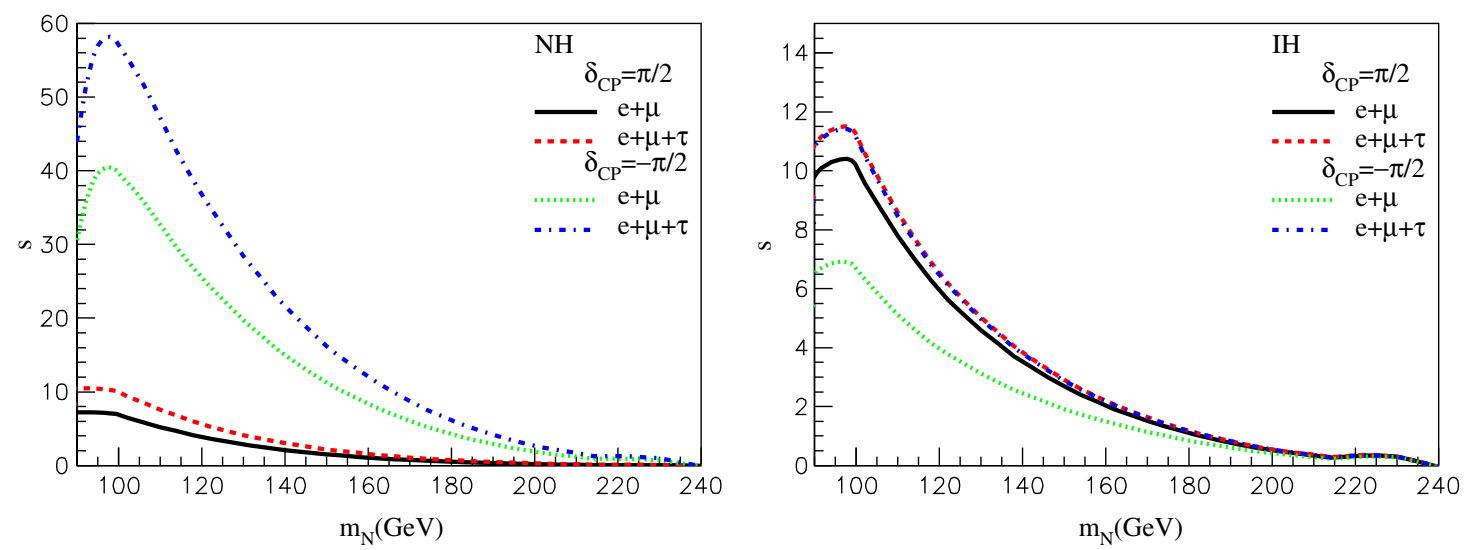

FIG. 10. The significance $s$ vs $m_{N}$ for NH (left) and IH (right) with integrated luminosity $5 \mathrm{ab}^{-1}$. We choose $e^{y}=1750, \delta_{\mathrm{CP}}= \pm \pi / 2$, $\phi_{1}=\phi_{2}=\phi_{3}=0$ for $\mathrm{NH}$ (the largest eigenvalue of the matrix of neutrino Yukawa couplings is $0.00173 \times \sqrt{m_{N}}$ ), and $e^{y}=350$, $\delta_{\mathrm{CP}}= \pm \pi / 2, \phi_{1}=\phi_{2}=\phi_{3}=0$ for IH (the largest eigenvalue of the matrix of neutrino Yukawa couplings is $0.000447 \times \sqrt{m_{N}}$ ).

$(1.61: 1: 1.32)$ for $\delta_{\mathrm{CP}}=\pi / 2\left(\delta_{\mathrm{CP}}=-\pi / 2\right)$. In the case of $\mathrm{NH}$ with $\delta_{\mathrm{CP}}=-\pi / 2$ and the case of IH with $\delta_{\mathrm{CP}}= \pm \pi / 2$, the three $\left|R_{l N}\right|$ are of similar magnitude, so $\left|R_{\mu N}\right|$ can be probed to order of $10^{-3}$ for $5 \mathrm{ab}^{-1}$ with an enhanced production rate due to large $\left|R_{e N}\right|$. In the case of $\mathrm{NH}$ with $\delta_{\mathrm{CP}}=\pi / 2,\left|R_{e N}\right|$ is almost 10 times smaller than $\left|R_{\mu N}\right|$, then the mixing $\left|R_{\mu N}\right|$ of order of $10^{-2}$ can be probed for $5 \mathrm{ab}^{-1}$,
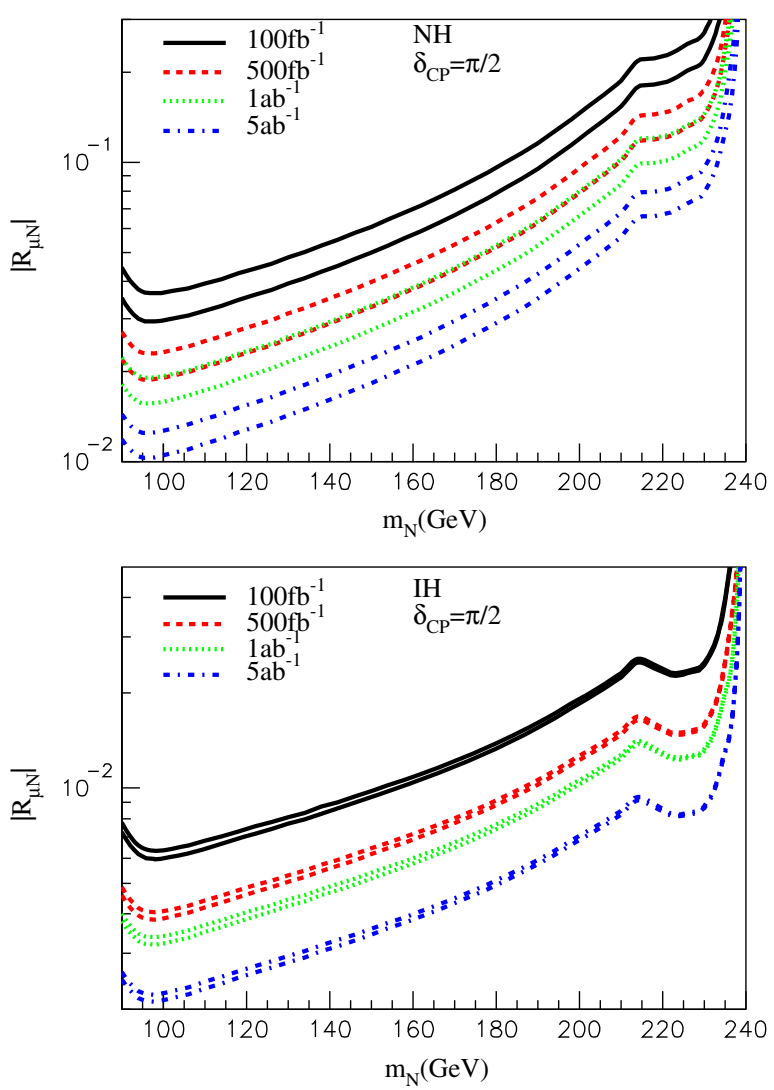

which is similar to the case with a single nonzero $\left|R_{\mu N}\right|$ as given in Fig. 6.

To conclude, in the low energy seesaw model, due to the correlation of three different $R_{l N}$, sizable $\left|R_{e N}\right|$ leads to t-channel production of heavy sterile neutrinos and can give rise to a quite large total production cross section of $e^{+} e^{-} \rightarrow N \nu$ process. The $N \rightarrow l j j$ events, on the other
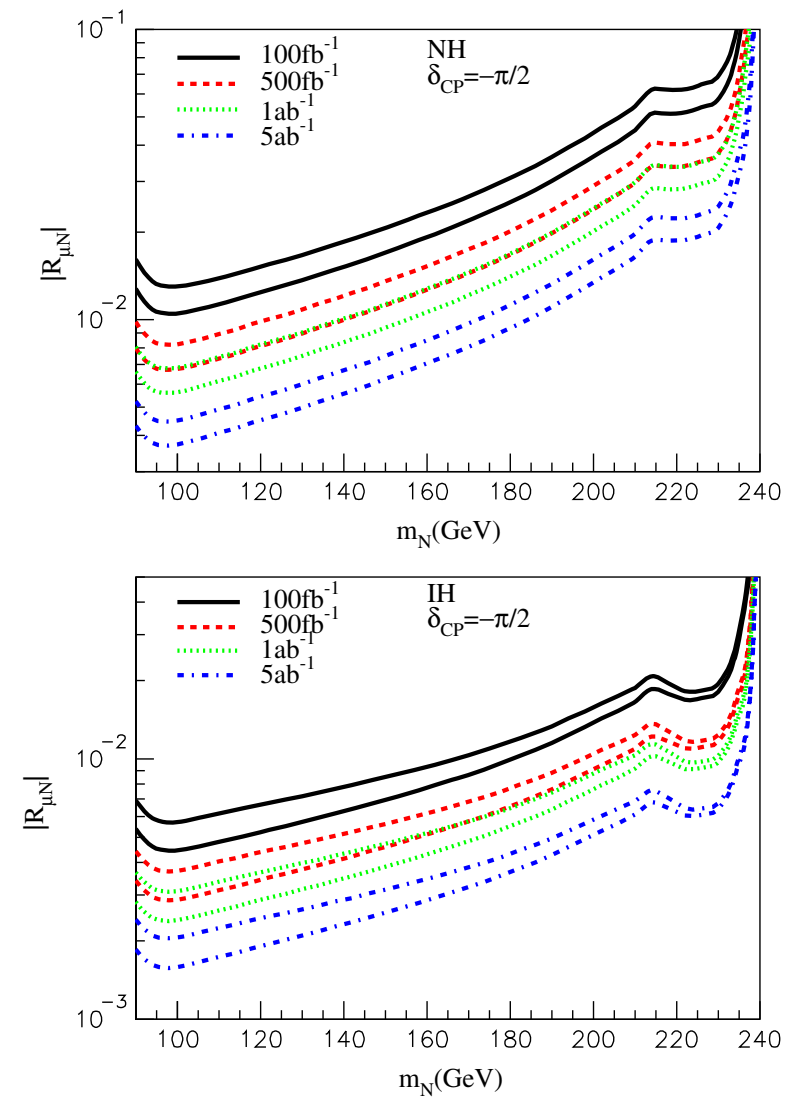

FIG. 11. Sensitivity to $\left|R_{\mu N}\right|$ with significance $s=5$ for the cases of NH and IH and Dirac phase $\delta_{\mathrm{CP}}= \pm \pi / 2, \phi_{1}=\phi_{2}=\phi_{3}=0$. The curves of different type in each plot from up to down correspond to integrated luminosities $100 \mathrm{fb}^{-1}, 500 \mathrm{fb}^{-1}, 1 \mathrm{ab}^{-1} \mathrm{and}^{5} \mathrm{ab}^{-1}$. The upper (lower) curve of the same type corresponds to $e+\mu(e+\mu+\tau)$ significances in each plot, respectively. 
hand, can be dominated by $\mu j j$ and $\tau j j$ events because $\left|R_{\mu N}\right|^{2}+\left|R_{\tau N}\right|^{2}$ can be much larger than $\left|R_{e N}\right|^{2}$ as can be seen in Fig. 1. For NH, in particular, $\left|R_{\mu N}\right|^{2}+\left|R_{\tau N}\right|^{2}$ is always much larger than $\left|R_{e N}\right|^{2}$. In this case, $e+\mu$ significance and $e+\mu+\tau$ significance can be quite different, as can be seen in the left panel of Fig. 8. On the other hand, for IH, $\left|R_{\mu N}\right|^{2}+\left|R_{\tau N}\right|^{2}$ can be of similar size of $\left|R_{e N}\right|^{2}$ and even much smaller than $\left|R_{e N}\right|^{2}$. In this case, the $e+\mu$ significance and $e+\mu+\tau$ significance would not be very different. This is the case for the right panel in Fig. 8. So analyzing the dominating of the $N$ decay channel and the difference between the $e+\mu$ and $e+\mu+\tau$ significances can give hints regarding the mass hierarchy of neutrinos. In particular, if the dominating $N \rightarrow l j j$ events are $e j j$ events, it has to be $\mathrm{IH}$.

\section{CONCLUSION}

In summary, we have studied the production, decay, and signature in $l j j$ events of heavy Majorana-type sterile neutrinos of mass around $100 \mathrm{GeV}$ at future CEPC. We study the tree-level decay of heavy sterile neutrinos by carefully taking into account the propagator of bosons, such as $W$ and $Z$. Effects of on-shell and off-shell $W$ and $Z$ bosons are all taken into account by including the width of $W$ and $Z$ in the propagators. We obtain an analytic formula for tree-level decay of heavy sterile neutrinos which is valid for mass from tens of $\mathrm{GeV}$ to hundreds of $\mathrm{GeV}$ and higher energy. The formula is valid, in particular, for mass $m_{N}$ around the masses of bosons.

For convenience and for later discussion in the low energy seesaw model of heavy sterile neutrinos, we have first studied the production of a single heavy sterile neutrino at CEPC and its signature. Although the mixing of a single heavy sterile neutrino with active neutrinos is strongly constrained by the $0 \nu \beta \beta$ experiment, the study of the signature of a single heavy sterile neutrino is also of interests in and of itself, since some other particles or mechanisms, e.g., extra scalars or type-II seesaw, may exist to ease the constraint. We have shown that for a single heavy sterile neutrino, an electron positron collider such as CEPC is more sensitive to the mixing of a heavy sterile neutrino with electron (anti)neutrinos, than the mixing with muon or tau (anti)neutrinos. For the former, the production of $N$ is associated with the production of an electron neutrino or antineutrino and can go through the t-channel. The cross section of the t-channel process can be two orders of magnitude larger than the cross section of the s-channel process which is responsible for probing the magnitude of the mixing with muon or tau (anti)neutrinos. We found that for an integrated luminosity $5 \mathrm{ab}^{-1}$, CEPC can reach a $5 \sigma$ sensitivity of $R_{e N}$, the mixing of the sterile neutrino with active neutrinos, to a value as small as $\left|R_{e N}\right|=10^{-3}$. For the mixing with muon and tau (anti)neutrinos $R_{\mu N}$ and $R_{\tau N}$, the $5 \sigma$ sensitivity can reach $\left|R_{\mu N, \tau N}\right| \approx 10^{-2}$.
We also study the production of heavy sterile neutrinos in a low energy seesaw model and their signature at CEPC. In this model, two heavy sterile neutrinos exist so that an explanation of the masses and mixings of active neutrinos is available using the seesaw mechanism. In this model, the mixings of these two heavy sterile neutrinos with active neutrinos, $R_{l N_{1}}$ and $R_{l N_{2}}$, are forced to have the same magnitude for all $l$, if we want these mixings to be large. In this case, the masses of these two sterile neutrinos are found to be degenerate or quasidegenerate if taking into account the constraint from the $0 \nu \beta \beta$ experiment.

Therefore, the signature of these two heavy sterile neutrinos is just the double of the signature of a single heavy sterile neutrino discussed above. The major difference compared with the case of a single heavy sterile neutrino is that the mixing $R_{l N_{1}}$ is no longer arbitrary for different $l$. Instead, values of $R_{l N_{1}}$ for different $l$ have some correlations. We take these facts into account. We find that the Dirac $C P$ phase $\delta_{\mathrm{CP}}$ in the PMNS mixing matrix of active neutrinos and Majorana phases affects the mixing $R_{l N}$, and changes the relative significance of $e j j, \mu j j$, and $\tau j j$ events. Thus, a search for all 3 lepton channels is helpful to constrain the model. With sizable $R_{e N}$, the significance of both $\mu$ and $\tau$ channels will be enhanced, and will further constrain $R_{\mu N}$ and $R_{\tau N}$ compared to the case with only a single mixing.

We further note that although our analysis is for CEPC running at $240 \mathrm{GeV}$, it can also be applied to ILC running at around $250 \mathrm{GeV}$ without much modification [43].

\section{ACKNOWLEDGMENTS}

This research is supported in part by the Natural Science Foundation of China (NSFC), Grants No. 11135009 and No. 11375065, and in part by the Shanghai Key Laboratory of Particle Physics and Cosmology, Grant No. 15DZ2272100. X. H. W. would like to thank Qi-Shu Yan for helpful discussions on MadGraph and CEPC.

\section{APPENDIX: HEAVY STERILE NEUTRINO DECAYS}

In this section we summarize the tree-level decay rate of sterile neutrinos decaying to three final fermions through interaction with the $Z$ and $W$ bosons induced by mixing with active neutrinos. Effects of on-shell and off-shell $Z$ and $W$ bosons are all taken into account by including the width of $W$ and $Z$ in the propagators. For example, for $N \rightarrow l_{1}^{-} l_{2}^{+} \nu_{l_{2}}$ and $l_{1} \neq l_{2}$, the decay rate is obtained as follows:

$$
\begin{aligned}
\Gamma\left(N \rightarrow l_{1}^{-} l_{2}^{+} \nu_{l_{2}}\right)= & \left|R_{l_{1} N}\right|^{2} \frac{G_{F}^{2} m_{N}}{\pi^{3}} \int_{0}^{\frac{m_{N}}{2}} d E_{1} \int_{\frac{m_{N}}{2}-E_{1}}^{\frac{m_{N}}{2}} d E_{2}\left|X_{W}\right|^{2} \\
& \times \frac{1}{2}\left(m_{N}-2 E_{2}\right) E_{2},
\end{aligned}
$$


where $X_{W}$ comes from the propagator of the $W$ boson and is

$$
X_{W}=\frac{m_{W}^{2}}{q^{2}-m_{W}^{2}+i \Gamma_{W} m_{W}},
$$

where $q^{2}=m_{N}^{2}-2 m_{N} E_{1}$ and $\Gamma_{W}$ is the total decay rate of $W . q=p-p_{1}$ is the four-momentum of the $W$ boson where $p$ and $p_{1}$ are the four-momenta of $N$ and $l_{1}$, respectively. Thus, $q^{2}=m_{N}^{2}-2 m_{N} E_{1}$ when considering the decay of $N$ at rest and neglecting the mass of $l_{1}$ with $E_{1}$ the energy of $l_{1}$. After performing integration in (A1), we can get a formula for the decay rate as a function of $m_{N}, m_{W}$, and $\Gamma_{W}$. Similarly, we can get formulas for other decays through $Z$ boson exchange.

In the following we summarize the results:

(1) For $N \rightarrow l_{1}^{-} l_{2}^{+} \nu_{l_{2}}, N \rightarrow l_{1}^{+} l_{2}^{-} \bar{\nu}_{l_{2}}$ and $l_{1} \neq l_{2}$

$$
\Gamma\left(N \rightarrow l_{1}^{-} l_{2}^{+} \nu_{l_{2}}\right)=\Gamma\left(N \rightarrow l_{1}^{+} l_{2}^{-} \bar{\nu}_{l_{2}}\right)=\left|R_{l_{1} N}\right|^{2} \frac{G_{F}^{2} m_{N}^{5}}{\pi^{3}} F_{N}\left(m_{N}, m_{W}, \Gamma_{W}\right),
$$

where $F_{N}$ is a dimensionless function and is given in (A13) below.

(2) For $N \rightarrow l^{-} q_{1} \bar{q}_{2}, N \rightarrow l^{+} \bar{q}_{1} q_{2}$

$$
\Gamma\left(N \rightarrow l^{-} q_{1} \bar{q}_{2}\right)=\Gamma\left(N \rightarrow l^{+} \bar{q}_{1} q_{2}\right)=\left|R_{l N}\right|^{2} \frac{G_{F}^{2} m_{N}^{5}}{\pi^{3}} N_{C} F_{N}\left(m_{N}, m_{W}, \Gamma_{W}\right)\left|K_{q_{1} q_{2}}\right|^{2} .
$$

$K_{q_{1} q_{2}}$ is the CKM matrix element in $\left(q_{1}, q_{2}\right)$ entry, and $N_{C}=3$ the number of color degrees of freedom of quarks.

(3) For $N \rightarrow l^{-} l^{+} \nu_{l}, N \rightarrow l^{+} l^{-} \bar{\nu}_{l}$

$$
\begin{aligned}
\Gamma\left(N \rightarrow l^{-} l^{+} \nu_{l}\right)= & \Gamma\left(N \rightarrow l^{+} l^{-} \bar{\nu}_{l}\right) \\
= & \left|R_{l N}\right|^{2} \frac{G_{F}^{2} m_{N}^{5}}{\pi^{3}}\left[F_{N}\left(m_{N}, m_{W}, \Gamma_{W}\right)+\left(C_{L}^{2}+C_{R}^{2}\right) F_{N}\left(m_{N}, m_{Z}, \Gamma_{Z}\right)\right. \\
& \left.+2 C_{L} F_{S}\left(m_{N}, m_{W}, \Gamma_{W}, m_{Z}, \Gamma_{Z}\right)\right]
\end{aligned}
$$

where $C_{L, R}$ is given in (A10), $F_{S}$ is a dimensionless function and is given below in (A15).

(4) For $N \rightarrow \nu_{l} \bar{l}^{\prime} l^{\prime}$ and $N \rightarrow \bar{\nu}_{l} l^{\prime} \bar{l}^{\prime}$

$$
\Gamma\left(N \rightarrow \nu_{l} \bar{l}^{\prime} l^{\prime}\right)=\Gamma\left(N \rightarrow \bar{\nu}_{l} l^{\prime} \bar{l}^{\prime}\right)=\left|R_{l N}\right|^{2} \frac{G_{F}^{2} m_{N}^{5}}{\pi^{3}}\left(C_{L}^{2}+C_{R}^{2}\right) F_{N}\left(m_{N}, m_{Z}, \Gamma_{Z}\right) .
$$

(5) For $N \rightarrow \nu_{l} q \bar{q}$ and $N \rightarrow \bar{\nu}_{l} \bar{q} q$

$$
\Gamma\left(N \rightarrow \nu_{l} \bar{l}^{\prime} l^{\prime}\right)=\Gamma\left(N \rightarrow \bar{\nu}_{l} l^{\prime} \bar{l}^{\prime}\right)=\left|R_{l N}\right|^{2} \frac{G_{F}^{2} m_{N}^{5}}{\pi^{3}} N_{C}\left[\left(C_{L}^{q}\right)^{2}+\left(C_{R}^{q}\right)^{2}\right] F_{N}\left(m_{N}, m_{Z}, \Gamma_{Z}\right),
$$

where $q=u, d, c, s, b$ for $m_{N}<2 m_{t}$ and $C_{L, R}^{q}$ is given in (A11) and (A12).

(6) For $N \rightarrow \nu_{l} \nu_{l^{\prime}} \bar{\nu}_{l^{\prime}}$ and $N \rightarrow \bar{\nu}_{l} \bar{\nu}_{l^{\prime}} \nu_{l^{\prime}}, l \neq l^{\prime}$

$$
\Gamma\left(N \rightarrow \nu_{l} \nu_{l^{\prime}} \bar{\nu}_{l^{\prime}}\right)=\Gamma\left(N \rightarrow \bar{\nu}_{l} \nu_{l^{\prime}} \bar{\nu}_{l^{\prime}}\right)=\left|R_{l N}\right|^{2} \frac{G_{F}^{2} m_{N}^{5}}{\pi^{3}} C_{\nu}^{2} F_{N}\left(m_{N}, m_{Z}, \Gamma_{Z}\right),
$$

where $C_{\nu}=1 / 2$.

(7) For $N \rightarrow \nu_{l} \nu_{l} \bar{\nu}_{l}$ and $N \rightarrow \bar{\nu}_{l} \bar{\nu}_{l} \nu_{l}$

$$
\Gamma\left(N \rightarrow \nu_{l} \nu_{l} \bar{\nu}_{l}\right)=\Gamma\left(N \rightarrow \bar{\nu}_{l} \nu_{l} \bar{\nu}_{l}\right)=\left|R_{l N}\right|^{2} \frac{G_{F}^{2} m_{N}^{5}}{\pi^{3}} 4 C_{\nu}^{2} F_{N}\left(m_{N}, m_{Z}, \Gamma_{Z}\right) .
$$


Couplings $C_{L}, C_{R}$, etc. which appear in expressions above, are given as

$$
\begin{array}{cc}
C_{L}=-\frac{1}{2}+\sin ^{2} \theta_{W}, & C_{R}=\sin ^{2} \theta_{W}, \\
C_{L}^{u}=\frac{1}{2}-\frac{2}{3} \sin ^{2} \theta_{W}, & C_{R}^{u}=-\frac{2}{3} \sin ^{2} \theta_{W}, \\
C_{L}^{u}=-\frac{1}{2}+\frac{1}{3} \sin ^{2} \theta_{W}, & C_{R}^{u}=\frac{1}{3} \sin ^{2} \theta_{W} .
\end{array}
$$

For mass $m_{N}, m_{X}$, and decay rate $\Gamma_{X}$, the function $F_{N}$ used above is

$$
\begin{aligned}
F_{N}\left(m_{N}, m_{X}, \Gamma_{X}\right)= & \frac{m_{X}^{4}}{96 m_{N}^{8}}\left\{-2 m_{N}^{2}\left(m_{N}^{2}-m_{X}^{2}\right)\right. \\
& +\left(A_{X}+C_{X} \Gamma_{X}^{2} m_{X}^{2}\right) \frac{1}{\Gamma_{X} m_{X}}\left[\arctan \left(\frac{m_{N}^{2}-m_{X}^{2}}{\Gamma_{X} m_{X}}\right)-\arctan \left(\frac{-m_{X}^{2}}{\Gamma_{X} m_{X}}\right)\right] \\
& \left.-\frac{1}{2}\left(B_{X}+2 \Gamma_{X}^{2} m_{X}^{2}\right) \ln \left(\frac{\Gamma_{X}^{2} m_{X}^{2}+\left(m_{N}^{2}-m_{X}^{2}\right)^{2}}{\Gamma_{X}^{2} m_{X}^{2}+m_{X}^{4}}\right)\right\}
\end{aligned}
$$

where

$$
A_{X}=\left(m_{N}^{2}-m_{X}^{2}\right)^{2}\left(m_{N}^{2}+2 m_{X}^{2}\right), \quad B_{X}=6\left(m_{N}^{2}-m_{X}^{2}\right) m_{X}^{2}, \quad C_{X}=3\left(m_{N}^{2}-2 m_{X}^{2}\right) .
$$

Function $F_{S}$ in (A5) is given as

$$
F_{S}=\frac{1}{m_{N}^{4}} \int_{0}^{\frac{m_{N}}{2}} d E_{1} \int_{\frac{m_{N}}{2}-E_{1}}^{\frac{m_{N}}{2}} d E_{2}\left(X_{W} X_{Z}^{*}+X_{W}^{*} X_{Z}\right) \frac{1}{2}\left(m_{N}-2 E_{2}\right) E_{2},
$$

where

$$
X_{Z}=\frac{m_{Z}^{2}}{q_{3}^{2}-m_{Z}^{2}+i \Gamma_{Z} m_{Z}}
$$

$q_{3}^{2}=m_{N}^{2}-2 m_{N} E_{3}$ with $E_{3}=m_{N}-E_{1}-E_{2}$ when considering the decay of $N$ at rest and neglecting the mass of final fermions. Equation (A15) can not be obtained as an explicit function of $m_{N}, m_{W}$, and $m_{Z}$. We can calculate this function numerically.

For the $N \rightarrow \nu H$ decay, the effect described here can be similarly obtained with the introduction of a function $F_{N}\left(m_{N}, m_{H}, \Gamma_{H}\right)$. For example, for $N \rightarrow \nu_{l} \bar{f} f$ and $N \rightarrow \bar{\nu}_{l} \bar{f} f$

$$
\Gamma\left(N \rightarrow \nu_{l} \bar{f} f\right)=\Gamma\left(N \rightarrow \bar{\nu}_{l} \bar{f} f\right)=\frac{g^{2} m_{N}^{7}\left|R_{l N}\right|^{2} y_{f}^{2}}{16 \pi^{3} m_{W}^{2} m_{H}^{4}} N_{f} F_{N}\left(m_{N}, m_{H}, \Gamma_{H}\right),
$$

where $y_{f}$ is the Yukawa coupling of the fermion $f, N_{f}=1$ for $f$ being a lepton and $N_{f}=3$ for $f$ being a quark. Interference of the $N$ decay through the $Z$ boson and $H$ boson vanishes. Since the Yukawa coupling to fermion $f$ is always small for $f=b, c, s, d, u$ and leptons, inclusion of $N$ decay through the neutral Higgs boson does not change significantly the signature of the sterile neutrino $N$ discussed in this article, as long as we are not going to concentrate on the signature of $N$ coming from $N \rightarrow \nu_{l} b \bar{b}$ and $N \rightarrow \bar{\nu}_{l} b \bar{b}$ decay.

In the low energy limit $m_{N}^{2} \ll m_{W}^{2}$, we have $\left|X_{W}\right| \approx\left|X_{Z}\right| \approx 1$, and the above equations of decay rate, (A3), (A4), (A5), (A6), (A7), (A8), (A9), can be simplified as follows:

(1) For $N \rightarrow l_{1}^{-} l_{2}^{+} \nu_{l_{2}}, N \rightarrow l_{1}^{+} l_{2}^{-} \bar{\nu}_{l_{2}}$, and $l_{1} \neq l_{2}$

$$
\Gamma\left(N \rightarrow l_{1}^{-} l_{2}^{+} \nu_{l_{2}}\right)=\Gamma\left(N \rightarrow l_{1}^{+} l_{2}^{-} \bar{\nu}_{l_{2}}\right)=\left|R_{l_{1} N}\right|^{2} \frac{G_{F}^{2} m_{N}^{5}}{192 \pi^{3}},
$$


(2) For $N \rightarrow l^{-} q_{1} \bar{q}_{2}, N \rightarrow l^{+} \bar{q}_{1} q_{2}$

$$
\Gamma\left(N \rightarrow l^{-} q_{1} \bar{q}_{2}\right)=\Gamma\left(N \rightarrow l^{+} \bar{q}_{1} q_{2}\right)=\left|R_{l N}\right|^{2} \frac{G_{F}^{2} m_{N}^{5}}{192 \pi^{3}} N_{C}\left|K_{q_{1} q_{2}}\right|^{2} .
$$

(3) For $N \rightarrow l^{-} l^{+} \nu_{l}, N \rightarrow l^{+} l^{-} \bar{\nu}_{l}$

$$
\Gamma\left(N \rightarrow l^{-} l^{+} \nu_{l}\right)=\Gamma\left(N \rightarrow l^{+} l^{-} \bar{\nu}_{l}\right)=\left|R_{l N}\right|^{2} \frac{G_{F}^{2} m_{N}^{5}}{192 \pi^{3}}\left[\left(1+C_{L}\right)^{2}+C_{R}^{2}\right],
$$

(4) For $N \rightarrow \nu_{l} \bar{l}^{\prime} l^{\prime}$ and $N \rightarrow \bar{\nu}_{l} l^{\prime} \bar{l}^{\prime}$

$$
\Gamma\left(N \rightarrow \nu_{l} \bar{l}^{\prime} l^{\prime}\right)=\Gamma\left(N \rightarrow \bar{\nu}_{l} l^{\prime} \bar{l}^{\prime}\right)=\left|R_{l N}\right|^{2} \frac{G_{F}^{2} m_{N}^{5}}{192 \pi^{3}}\left(C_{L}^{2}+C_{R}^{2}\right) .
$$

(5) For $N \rightarrow \nu_{l} q \bar{q}$ and $N \rightarrow \bar{\nu}_{l} \bar{q} q$

$$
\Gamma\left(N \rightarrow \nu_{l} \bar{l}^{\prime} l^{\prime}\right)=\Gamma\left(N \rightarrow \bar{\nu}_{l} l^{\prime} \bar{l}^{\prime}\right)=\left|R_{l N}\right|^{2} \frac{G_{F}^{2} m_{N}^{5}}{192 \pi^{3}} N_{C}\left[\left(C_{L}^{q}\right)^{2}+\left(C_{R}^{q}\right)^{2}\right] .
$$

(6) For $N \rightarrow \nu_{l} \nu_{l^{\prime}} \bar{\nu}_{l^{\prime}}$ and $N \rightarrow \bar{\nu}_{l} \bar{\nu}_{l^{\prime}} \nu_{l^{\prime}}, l \neq l^{\prime}$

$$
\Gamma\left(N \rightarrow \nu_{l} \nu_{l^{\prime}} \bar{\nu}_{l^{\prime}}\right)=\Gamma\left(N \rightarrow \bar{\nu}_{l} \nu_{l^{\prime}} \bar{\nu}_{l^{\prime}}\right)=\left|R_{l N}\right|^{2} \frac{G_{F}^{2} m_{N}^{5}}{192 \pi^{3}} C_{\nu}^{2},
$$

(7) For $N \rightarrow \nu_{l} \nu_{l} \bar{\nu}_{l}$ and $N \rightarrow \bar{\nu}_{l} \bar{\nu}_{l} \nu_{l}$

$$
\Gamma\left(N \rightarrow \nu_{l} \nu_{l} \bar{\nu}_{l}\right)=\Gamma\left(N \rightarrow \bar{\nu}_{l} \nu_{l} \bar{\nu}_{l}\right)=\left|R_{l N}\right|^{2} \frac{G_{F}^{2} m_{N}^{5}}{192 \pi^{3}} 4 C_{\nu}^{2} .
$$

In all these results, the masses of the final fermions have all been neglected.

[1] P. Minkowski, Phys. Lett. B 67, 421 (1977); T. Yanagida, in Proceedings of the Workshop on the Unified Theory and the Baryon Number in the Universe, edited by O. Sawada and A. Sugamoto (KEK Collaboration, Tsukuba, 1979), p. 95; M. Gell-Mann, P. Ramond, and R. Slansky, in Supergravity, edited by P. van Nieuwenhuizen and D. Freedman (NorthHolland, Amsterdam, 1979), p. 315; S. L. Glashow, in Proceedings of the 1979 Cargese Summer Institute on Quarks and Leptons, edited by M. Levy et al. (Plenum Press, New York, 1980), p. 687; R. N. Mohapatra and G. Senjanovic, Phys. Rev. Lett. 44, 912 (1980).

[2] T. Asaka and M. Shaposhnikov, Phys. Lett. B 620, 17 (2005); T. Asaka, S. Blanchet, and M. Shaposhnikov, Phys. Lett. B 631, 151 (2005).

[3] X.-G. He, T. Li, and W. Liao, Phys. Rev. D 81, 033006 (2010).

[4] W. Liao, Phys. Rev. D 82, 073001 (2010).

[5] M. Acciarri et al. (L3 Collaboration), Phys. Lett. B 461, 397 (1999).

[6] P. Achard et al. (L3 Collaboration), Phys. Lett. B 517, 67 (2001).
[7] F. del Aguila, E. Laermann, and P. M. Zerwas, Nucl. Phys. B297, 1 (1988).

[8] W. Buchmuller and C. Greub, Nucl. Phys. B363, 345 (1991).

[9] J. Gluza and M. Zralek, Phys. Rev. D 48, 5093 (1993).

[10] A. Djouadi, Z. Phys. C 63, 317 (1994).

[11] G. Azuelos and A. Djouadi, Z. Phys. C 63, 327 (1994).

[12] R. Vuopionpera, Z. Phys. C 65, 311 (1995).

[13] J. Gluza and M. Zralek, Phys. Lett. B 372, 259 (1996).

[14] J. Gluza and M. Zralek, Phys. Rev. D 55, 7030 (1997).

[15] A. Hofer and L. M. Sehgal, Phys. Rev. D 54, 1944 (1996).

[16] J. Gluza, J. Maalampi, M. Raidal, and M. Zralek, Phys. Lett. B 407, 45 (1997).

[17] G. Cvetic, C. S. Kim, and C. W. Kim, Phys. Rev. Lett. 82, 4761 (1999).

[18] F. del Aguila, J. A. Aguilar-Saavedra, A. Martinez de la Ossa, and D. Meloni, Phys. Lett. B 613, 170 (2005).

[19] A. Das and N. Okada, Phys. Rev. D 88, 113001 (2013).

[20] A. Blondel et al. (FCC-ee study Team), Nucl. Part. Phys. Proc. 273-275, 1883 (2016). 
[21] S. Banerjee, P. S. B. Dev, A. Ibarra, T. Mandal, and M. Mitra, Phys. Rev. D 92, 075002 (2015).

[22] A. Caputo, P. Hernandez, M. Kekic, J. López-Pavón, and J. Salvado, Eur. Phys. J. C 77, 258 (2017).

[23] S. Antusch, E. Cazzato, and O. Fischer, J. High Energy Phys. 12 (2016) 007.

[24] S. Antusch, E. Cazzato, and O. Fischer, Int. J. Mod. Phys. A 32, 1750078 (2017).

[25] S. S. Biswal and P. S. B. Dev, Phys. Rev. D 95, 115031 (2017).

[26] C. X. Yue, Y. C. Guo, and Z. H. Zhao, Nucl. Phys. B925, 186 (2017).

[27] CEPC-SppC Preliminary Conceptual Design Report, http:// cepc.ihep.ac.cn/preCDR/volume.html.

[28] P. Benes, A. Faessler, F. Simkovic, and S. Kovalenko, Phys. Rev. D 71, 077901 (2005).

[29] K. A. Olive et al. (Particle Data Group), Chin. J. Phys. C 38, 090001 (2014).

[30] S. Antusch, C. Biggio, E. Fernandez-Martinez, M. B. Gavela, and J. Lopez-Pavon, J. High Energy Phys. 10 (2006) 084.

[31] S. Antusch, J. P. Baumann, and E. Fernandez-Martinez, Nucl. Phys. B810, 369 (2009).

[32] A. Ibarra, E. Molinaro, and S. T. Petcov, Phys. Rev. D 84, 013005 (2011).
[33] M. Drewes and B. Garbrecht, Nucl. Phys. B921, 250 (2017).

[34] A. de Gouva and A. Kobach, Phys. Rev. D 93, 033005 (2016).

[35] E. Fernandez-Martinez, J. Hernandez-Garcia, and J. LopezPavon, J. High Energy Phys. 08 (2016) 033.

[36] J. Alwall, R. Frederix, S. Frixione, V. Hirschi, F. Maltoni, O. Mattelaer, H.-S. Shao, T. Stelzer, P. Torrielli, and M. Zaro, J. High Energy Phys. 07 (2014) 079.

[37] A. Alloul, N. D. Christensen, C. Degrande, C. Duhr, and B. Fuks, Comput. Phys. Commun. 185, 2250 (2014).

[38] C. Degrande, C. Duhr, B. Fuks, D. Grellscheid, O. Mattelaer, and T. Reiter, Comput. Phys. Commun. 183, 1201 (2012).

[39] A. Atre, T. Han, S. Pascoli, and B. Zhang, J. High Energy Phys. 05 (2009) 030.

[40] F. del Aguila and J. A. Aguilar-Saavedra, Nucl. Phys. B813, 22 (2009).

[41] T. Sjostrand, S. Mrenna, and P. Z. Skands, J. High Energy Phys. 05 (2006) 026.

[42] J. Conway, Pretty Good Simulator webpage, http://conway .physics.ucdavis.edu/research/software/pgs/pgs4-general .htm.

[43] K. Fujii et al., arXiv:1710.07621. 